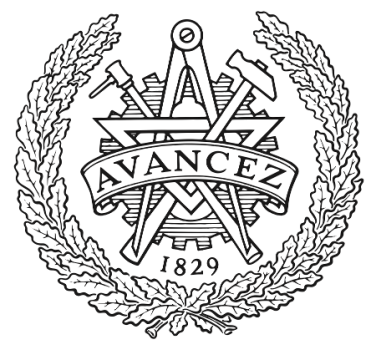

CHALMERS

UNIVERSITY OF TECHNOLOGY

\title{
Analysis of possible improvement of the plasma performance in JET due to the inward spatial channelling of fast-ion energy
}

Downloaded from: https://research.chalmers.se, 2023-04-26 09:08 UTC

Citation for the original published paper (version of record):

Kolesnichenko, Y., Lutsenko, V., Tyshchenko, M. et al (2018). Analysis of possible improvement of the plasma performance in JET due to the inward spatial channelling of fast-ion energy. Nuclear Fusion, 58(7). http://dx.doi.org/10.1088/1741-4326/aac09f

N.B. When citing this work, cite the original published paper. 
PAPER

Analysis of possible improvement of the plasma performance in JET due to the inward spatial channelling of fast-ion energy

To cite this article: Ya.I. Kolesnichenko et al 2018 Nucl. Fusion 58076012

View the article online for updates and enhancements.
You may also like

- Accurate and efficient calculation of
photoionization in streamer discharges
$\frac{\text { using fast multipole method }}{\text { Bo Lin, Chijie Zhuang, Zhenning Cai et al. }}$
- Comparative performance of the finite
$\frac{\text { element method and the boundary }}{\text { element fast multipole method for }}$
problems mimicking transcranial magnetic
stimulation (TMS)
Aung Thu Htet, Guilherme B Saturnino,
Edward H Burnham et al.
- Comparison of absorption simulation in
$\frac{\text { semiconductor nanowire and nanocone }}{\text { arrays with the Fourier modal method, the }}$
$\frac{\text { finite element method, and the finite- }}{\text { difference time-domain method }}$
Nicklas Anttu, Henrik Mäntynen, Toufik
Sadi et al.




\title{
Analysis of possible improvement of the plasma performance in JET due to the inward spatial channelling of fast-ion energy
}

\author{
Ya.I. Kolesnichenko ${ }^{1}$, V.V. Lutsenko ${ }^{1}$, M.H. Tyshchenko ${ }^{1}$, H. Weisen ${ }^{2}$, \\ Yu.V. Yakovenko ${ }^{1}$ and JET Contributors ${ }^{\mathrm{a}}$ \\ ${ }^{1}$ Institute for Nuclear Research, Prospekt Nauky 47, Kyiv 03680, Ukraine \\ 2 Swiss Plasma Center, École Polytechnique Fédérale de Lausanne, Switzerland \\ E-mail: yk@kinr.kiev.ua
}

Received 3 January 2018, revised 18 April 2018

Accepted for publication 27 April 2018

Published 15 May 2018

\begin{abstract}
Effects of the spatial chanelling (SC) of the energy of fusion-produced alpha particlesthe spatial transfer of the energy of fast ions by destabilized eigenmodes and delivering this energy to bulk plasma particles (Kolesnichenko et al 2010 Phys. Rev. Lett. 104 075001) — on the plasma performance is studied. Analysis is carried out in the assumption that alpha particles located in the peripheral region of the plasma destabilize multiple fast magnetoacoustic modes (FMM) having global radial structure. The FMM with the frequencies close to cyclotron harmonics of alpha particles are considered. It is found that these FMM can be in resonance with the bulk plasma ions and electrons located in the central region of the plasma, delivering the alpha energy to this region. This improves the overall plasma confinement. In addition, it leads to anomalous ion heating when the ion damping of FMM exceeds the electron one. The damping rates of the considered waves are calculated. It is shown that reasonably small amplitude waves can receive and transfer across the flux surfaces as large power density as that required for spatial channelling of a considerable part of fusion energy. The developed theory of the inward spatial channelling is applied to JET experiments carried out during the deuterium-tritium-experiment campaign (DTE1), where presumably anomalous ion heating and improvement of the plasma confinement took place.
\end{abstract}

Keywords: tokamaks, energetic ions, alpha particles, waves, eigenmodes, instabilities

(Some figures may appear in colour only in the online journal)

\section{Introduction}

Plasma heating is often accompanied by degradation of its energy confinement time. However, it seems that the overall confinement time was not less but slightly higher at the largest fusion power in deuterium-tritium (DT) experiments on JET during the deuterium-tritium-experiment campaign (DTE1), see [1-3] and appendix A.

\footnotetext{
${ }^{\text {a }}$ See the author list of [40].
}

The same experiments have shown, in addition, that the central ion temperature in DT discharges was higher than that in deuterium (D) discharges where the ion cyclotron resonance heating (ICRH) was applied with the heating power equal to the power of alpha particles (fusion products) in DT discharges. This fact indicates the presence of some anomalous heating mechanism because mainly electrons are heated during slowing down of $3.5 \mathrm{MeV}$ alpha particles by Coulomb collisions.

The improvement of the plasma confinement due to thermonuclear reaction would be of practical importance in a 
future DT experiment on JET [4] and for ITER. The scenarios with $T_{i}>T_{e}\left(T_{i}\right.$ and $T_{e}$ are the ion temperature and electron temperature, respectively) may be also of importance because they provide the maximum fusion reactivity for given plasma density and $\beta$ (the ratio of the plasma pressure to the magnetic field pressure).

This motivated us to carry out this work. Its purpose is to see whether the spatial channelling (SC) of the energy and momentum of fast ions - the phenomenon predicted in [5], see also [6] — can improve the plasma performance and, in particular, explain the mentioned observations in JET.

The SC is the transfer of the energy and momentum of fast ions by the modes destabilized by these ions from one region to another one. It takes place when the region where fast ions transfer their energy to the modes and the region where these modes are damped due to interaction with the bulk plasma do not coincide.

According to [5], the SC can explain experiments in the NSTX spherical torus, where a strong increase (by a factor of three) of the neutral beam injection (NBI) heating power resulted in broadening of the temperature profile, but not in growth of the temperature in the plasma core. In these experiments described in [7], an Alfvénic activity with multiple frequencies in the range $(0.2-0.5) \omega_{B i}$ ( $\omega_{B i}$ is the ion gyrofrequency, with the second symbol in the subscript indicating the species to which the gyrofrequency $\omega_{B}$ refers) was observed. Taking this into account, it was assumed in [5] that the fast ions located in the core region (at $r \sim r_{f}$, where $r_{f}$ is a radius in the plasma core, $r_{f} \ll a, a$ is the plasma radius) were responsible for the destabilization of Alfvén eigenmodes, which were damped at the plasma periphery (at $r \sim r_{\text {damp, }}$, with $r_{\text {damp }}>r_{f}$ ). This explained the negative effect of the SC on the plasma heating.

Note that an alternative explanation of the mentioned NSTX experiments was that destabilized multiple Alfvén modes resulted in the stochastization of electron drift orbits and concomitant anomalous electron thermal diffusivity, $\kappa_{e}$ [8]. However, recently it was concluded that neither global Alfvén eigenmodes (GAE) nor compressional Alfvén eigenmodes (CAE, another name of the CAE is fast magnetoacoustic modes, FMM) have visible effects on $\kappa_{e}$ : it was found that the measured wave amplitudes were not sufficient to produce stochasticity, although they were not small (with density fluctuation amplitudes of the order of $10^{-4}-10^{-3}$ for CAEs and $10^{-5}-10^{-4}$ for GAEs) [9].

The effect of the SC would be positive if the modes were damped in the near-axis region while an instability were driven at larger radii, $r \sim r_{\text {damp }} \ll a$ and $r_{\text {damp }}<r_{f} \lesssim a$. This is our basic idea which will be verified in the work. We will consider whether FMM destabilized by fusion-produced alpha particles can lead to improvement of the plasma confinement by transferring the fusion energy to the near-axis region. In other words, we will consider inward spatial channelling.

Our basic idea is illustrated in figure 1 .

The structure of the work is the following. Section 2 is aimed to give an answer to the question whether FMM can interact resonantly with fusion produced alpha particles in the peripheral plasma region and bulk plasma particles in the
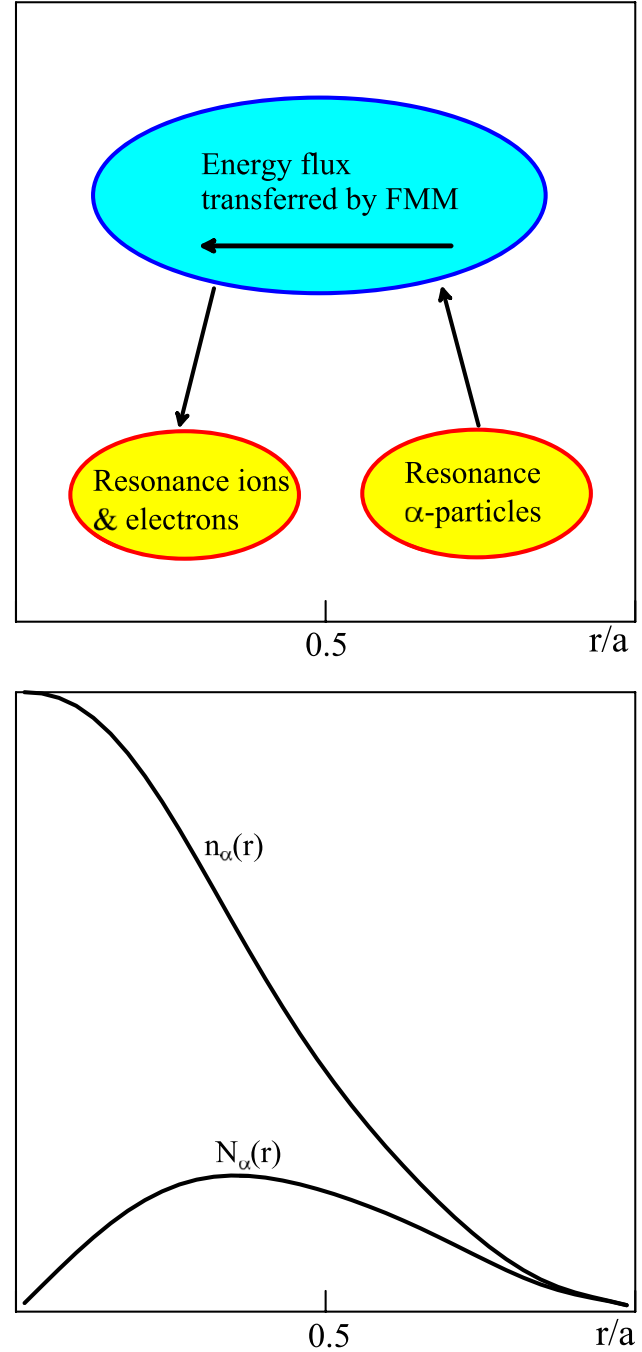

Figure 1. Sketch demonstrating inward SC of alpha energy: lower panel, radial distributions of the $\alpha$-particle density and the number of alphas on flux surfaces $\left(N_{\alpha}(r)=r n_{\alpha}(r)\right)$. Upper panel, the energy transferred from the resonance $\alpha$-particles located in the peripheral region to the bulk plasma ions and electrons via FMM. Arrows show directions of energy fluxes.

plasma core, which is a necessary condition for the inward SC based on FMM. Section 3 considers two other principal issues of the spatial energy transfer: first, the wave amplitudes required for the $\mathrm{SC}$ of a considerable part of fusion energy and, second, the efficiency of the transfer of this energy by FMM across the magnetic field; in addition, conditions required for persistence of the eigenmode structure during instabilities are considered. The effect of the SC on the plasma central temperature is studied in a one-fluid model in section 4 . In the subsequent two sections we consider possibilities to obtain the ion temperature exceeding the electron one: At the beginning, in section 5, a general analysis is carried out. In section 6 , specific numerical calculations employing JET parameters are carried out. In section 7 the results obtained are summarized and open questions are discussed. Appendix A contains an analysis of JET discharges with DT plasmas, where presumably the anomalous ion heating was observed and improvement of the plasma confinement took place. These discharges 
are compared with deuterium plasma discharges in the presence and in the absence of ICRH. The fraction of alpha particles with the birth energy in the peripheral part of the plasma is investigated in appendix B; the analysis carried out includes effects of finite orbit width of the particles. The damping rates of fast magnetoacoustic waves due to their resonance interaction with the bulk plasma ions and electrons are calculated in appendix $\mathrm{C}$.

\section{Resonances of FMM with alpha particles and thermal particles}

Fast magnetoacoustic modes were destabilized in many experiments on tokamaks. It seems, FMM instabilities were responsible for superthermal ion cyclotron emission (ICE) observed in JET [10], TFTR [11], JT-60 [12], LHD [13], DIII-D [14], ASDEX Upgrade [15] and other devices. In particular, in the JET Preliminary Tritium Experiment (PTE) the ICE power spectrum had sharp peaks at the frequencies $\omega=l \omega_{B \alpha}^{\min }$, with $l<7$; at higher frequencies, $\omega>7 \omega_{B \alpha}$, the spectrum was continuous, $98 \%$ of the emitted power being associated with this part of the spectrum [16]. Here and below the subscript ' $\alpha$ ' labels alpha particles, the superscript 'min' means that the value is taken in a point near the outer circumference of the torus, where the magnetic field $(B)$ is minimum and where presumably the source of the ICE detected was located, and $l$ is an integer. The ICE associated with the energetic ions arising during ICRH (accelerated H-minority in D-plasma) was also observed in JET [17], see a more recent paper [18].

Theoreticians explain the ICE observed in the most experiments by the resonance excitation of FMM by energetic ions, see e.g. [19-22, 24] and overview [23]. Fast FMM instabilities (with the growth rates exceeding inverse bounce/transit time of the energetic ions, $\left.\gamma>\left(\tau_{b \alpha}\right)^{-1}\right)$ and slow instabilities (with $\gamma<\left(\tau_{b \alpha}\right)^{-1}$ ) are considered. The cyclotron resonance is responsible for the instabilities in all the ICE theories. Only local resonances take place during fast instabilities, whereas both local resonances and global resonances (the resonances containing bounce averaged magnitudes) are present in slow instabilities.

Below we consider local resonances between the FMM and particles (alpha particles, bulk plasma ions, and electrons).

The local ion cyclotron resonance is given by

$$
\omega-l \omega_{B}(x)-\omega_{D}(r, \vartheta)=k_{\|}(r) v_{\|}
$$

where $\omega$ is the mode frequency, $\omega_{B}=\bar{\omega}_{B}(1-x / R)$, $\bar{\omega}_{B}=\omega_{B}(r=0), R$ is the major radius of the torus, $x=r \cos \vartheta$, $r$ and $\vartheta$ are the radial coordinate and poloidal coordinate, respectively, $\omega_{D}$ is the toroidal drift frequency, $v_{\|}$is the particle velocity along the magnetic field, $k_{\|}=(m-n q) /(q R)$ is the longitudinal wavenumber, $m$ and $n$ are the mode numbers (poloidal and toroidal).

In the region close to the outer edge of the torus (where the ICE source is located) the FMM instability growth rate is maximum when $k_{\|} v_{\| \alpha}<\omega_{D \alpha}$ [21, 22]. The instability is driven by the velocity anisotropy of the alpha distribution function, which arises because of large orbit width of alphas.
The question we want to clarify is whether FMM with $\omega>\omega_{B \alpha}$ can transfer the alpha energy to the bulk plasma ions located close to the magnetic axis.

We assume that multiple high frequency FMM occupy a considerable part of the plasma cross section, rather than being localized at the plasma periphery where the source of ICE is located. Recent measurements of the FMM with $\omega \lesssim \omega_{B i}$ in the NSTX spherical torus [25] and a numerical calculation [26] support this assumption (see also [27]). However, till now there were no experimental measurements of the radial structure of high-frequency FMM in JET and, to our knowledge, in other tokamaks. Hopefully, forthcoming experiments on JET will give an answer on the structure of FMM with the frequencies above $20 \mathrm{MHz}$ (the gyrofrequency of alpha particles and deuterons at $B=3.5 \mathrm{~T}$ is $26.7 \mathrm{MHz}$ ).

Different locations of the modes with various mode numbers, as well as finite width of their resonances with energetic ions, lead to a continuous frequency spectrum of the FMM instabilities, although the mode numbers are discrete. As we already mentioned, this kind of the frequency spectrum contained almost all the total integrated ICE emission power in the JET PTE [16]. Therefore, we will not restrict our consideration to FMM with the frequencies $l \omega_{B i}^{\min }$. Instead, when analyzing the resonances, we will assume that $l \omega_{B \alpha}^{\min } \leqslant \omega \leqslant l \bar{\omega}_{B \alpha}$ (with $\bar{\omega}_{B \alpha}=\omega_{B \alpha}(r=0)$ ) and take

$$
\omega=l_{\alpha} \bar{\omega}_{B \alpha}\left(1-r_{1} / R\right),
$$

with $r_{1}$ a certain radius, $0 \leqslant r_{1} \leqslant a$.

\subsection{The case of $k_{\| \alpha} v_{\| \alpha} \gg \omega_{D \alpha}$}

Applying the resonance equation (1) to alphas and plasma ions, we obtain:

$$
\frac{v_{\| i}^{\mathrm{res}}}{v_{\| \alpha}}=\frac{k_{\| \alpha}}{k_{\| i}} \frac{\omega-l_{i} \bar{\omega}_{B i}+l_{i} \bar{\omega}_{B i} x_{i} / R-\omega_{D i}}{\omega-\bar{\omega}_{B \alpha}+l_{\alpha} \bar{\omega}_{B \alpha} x_{\alpha} / R-\omega_{D \alpha}},
$$

where $\left(r_{i}, x_{i}\right)$ is a point where a plasma ion with the velocity $v_{\| i}^{\text {res }}$ is in the resonance with the wave. In this subsection, we consider the case of $k_{\| \alpha} v_{\| \alpha} \gg \omega_{D \alpha}$ for alpha particles and a similar assumption is made for thermal ions, in order to neglect drift terms.

We assume first that $l_{i}=l_{\alpha}$. In this case the largest terms $\left(\sim l \omega_{B}\right)$ in equation (3) compensate each other for deuterons (because $\bar{\omega}_{B i}=\bar{\omega}_{B \alpha}$ ). Due to this, equation (3) is reduced to

$$
\frac{v_{\| i}^{\mathrm{res}}}{v_{\| \alpha}}=\frac{k_{\| \alpha} \tilde{r}_{i}}{k_{\| i} \tilde{r}_{\alpha}}
$$

where $\tilde{r}_{\alpha} \equiv x_{\alpha}-r_{1} \neq 0, \tilde{r}_{i} \equiv x_{i}-r_{1} \neq 0$. We observe that $v_{\| i}^{\text {res }} \ll v_{\| \alpha}$ when $\tilde{r}_{i} \ll \tilde{r}_{\alpha}$, unless $k_{\| \alpha} / k_{\| i}$ is large. Thus, this simple relation demonstrates that, in principle, the same FMM can simultaneously be in the resonance with both the peripheral high-energy alpha particles and near-axis thermal deuterons.

However, a more detailed analysis is required to see whether $v_{\| i}^{\text {res }}$ satisfies certain requirements. The matter is that the SC from the periphery to the plasma center can be efficient when two conditions are satisfied: (i) in the plasma periphery, 
the wave damping should be negligible, (ii) in the plasma core, it should be considerable but not too large, not preventing an FMM instability (the instability growth rate $\gamma \equiv \gamma_{\alpha}-\gamma_{d} \geqslant 0$, with $\gamma_{\alpha}$ and $\gamma_{d}$ are the instability drive caused by alphas and the wave damping, respectively). This implies that the ratio $v_{\| i}^{\text {res }} / v_{T i}\left(v_{T i}=\sqrt{2 T_{i} / M_{i}}\right.$ is the ion thermal velocity) should be very large in the periphery but moderately large in the core.

In order to find $v_{\| i}^{\text {res }}$, we use the resonance equation for the ions:

$$
\omega-l_{i} \bar{\omega}_{B i}\left(1-\frac{x_{i}}{R}\right)=k_{\| i} v_{\| i}^{\mathrm{res}},
$$

where the cyclotron harmonic number $l_{i}$ can differ from $l_{\alpha}$. We take $l_{i}=l_{\alpha}-j$, with $j=0, \pm 1, \ldots,|j| \ll l_{\alpha}$ : Eliminating $\omega$ from (5) by means of (2), we obtain for deuterons:

$$
v_{\| i}^{\mathrm{res}}=\frac{l_{\alpha} \bar{\omega}_{B i}}{k_{\| i} R}\left(\tilde{r}_{i}+\frac{j}{l_{\alpha}} R\right),
$$

where $k_{\| i}$ is to be specified. One can see that the resonance velocity of tritons is considerably larger than that given by (6) (because the main terms in the numerator of equation (3) do not compensate each other for tritons, $\bar{\omega}_{B \alpha} \neq \bar{\omega}_{B i}$ ).

The longitudinal wavenumber of unstable waves is determined by the alpha resonance condition. It is given by

$$
k_{\| \alpha}=\frac{l \bar{\omega}_{B \alpha} \tilde{r}_{\alpha}}{v_{\| \alpha} R}
$$

Knowing $k_{\| \alpha}$, we can calculate $k_{\| i}$ by means of the following general relationship:

$$
\frac{k_{\| i}}{k_{\| \alpha}}=1+\frac{m\left(\iota_{i}-\iota_{\alpha}\right)}{k_{\| \alpha} R} .
$$

It is clear that $k_{\| \alpha} \neq k_{\| i}$ because of the magnetic shear. However, when the second term in (8) is small, $k_{\| \alpha} \approx k_{\| i}$. This is the case for

$$
\Delta \iota \equiv\left|\iota_{i}-\iota_{\alpha}\right| \ll \frac{\tilde{r}_{\alpha}}{r_{*}} \frac{v_{A *}}{\left|v_{\| \alpha}\right|} \frac{k_{*}}{\left|k_{\vartheta}\right|},
$$

where $r_{*}$ is defined by the relation $\omega=k_{*} v_{A *}$, and $\left|k_{\vartheta}\right|=|m| / r_{*}<k_{*}, \quad|m|=\left(r_{*} \omega / v_{A *}\right)\left(\left|k_{\vartheta}\right| / k_{*}\right)$. The second term in (8), if not small, considerably increases the ratio of $k_{\| i} / k_{\| \alpha}$ or decreases it, depending on the sign of $m$.

An estimate of the ion damping rate made in appendix $\mathrm{C}$ shows that the required magnitude of the ion resonance velocity in JET lies in the range $v_{\| i}^{\text {res }} / v_{T i}=1.5-2$. Waves with lower $v_{\| i}^{\text {res }} / v_{T i}$ will not be excited, waves with higher velocities will not interact with the ions.

The requirement $v_{\| i}^{\text {res }} / v_{T i}=1.5-2$ does not contradict to equation (6) with $r_{i} \ll r_{\alpha}$. To see it, we write (6) in the form:

$$
\frac{x_{\alpha}}{a}=\frac{r_{1}}{a}+w\left(\frac{x_{i}}{a}-\frac{r_{1}}{a}-\frac{j A}{l}\right),
$$

where $w=\chi_{\alpha}\left(v_{\alpha} / v_{T i}\right)\left(v_{T i} / v_{\| i}^{\text {res }}\right)\left(k_{\| \alpha} / k_{\| i}\right), \chi=v_{\|} / v, w>0$ or $w<0, x_{i}=r_{i} \cos \vartheta_{i}$ and $x_{\alpha}=r_{\alpha} \cos \vartheta_{\alpha}$. For instance, when $T_{i 0} \approx 20 \mathrm{keV},\left|\chi_{\alpha}\right|=0.5,\left|v_{\| i}^{\text {res }}\right| / v_{T i}=1.5, k_{\| i}=k_{\| \alpha}, j=0$ we obtain $|w|=3.3, r_{\alpha}: r_{1}: r_{i}=5.3: 2: 1$ for $w=-3.3$. Moreover, due to $j \neq 0$ resonance, there is a solution with $x_{i} \ll r_{1} \approx x_{\alpha}$ at any ratio of $k_{\| i} / k_{\| \alpha}$. The condition for this is $r_{1} / a \approx j A / l_{\alpha}$, where $A=R / a$ is the aspect ratio of the torus. For instance, when $A=3.3, j=1$, this condition yields $r_{1} / a=0.82$ for $l_{\alpha}=4\left(l_{i}=3\right)$ and $r_{1} / a=0.66$ for $l_{\alpha}=5$ $\left(l_{i}=4\right)$.

The SC delivers the alpha energy not only to the ions but also to electrons. The condition of small electron damping is

$$
\frac{\omega^{2}}{k_{\|}^{2}(r) v_{T e}^{2}}=\left(\frac{R v_{\| \alpha}}{\tilde{r}_{\alpha} v_{T e}(r)} \frac{k_{\| \alpha}}{k_{\|}(r)}\right)^{2} \gg 1,
$$

where $v_{T e}=\sqrt{2 T_{e} / M_{e}}$ is the electron thermal velocity. This can be written as

$$
\frac{r_{\alpha}}{a}<\frac{A\left|\chi_{\alpha}\right| v_{\alpha}}{\left(1-r_{1} / r_{\alpha}\right) v_{T e}}\left|\frac{k_{\| \alpha}}{k_{\|}(r)}\right| .
$$

For the parameters used above $\left(A=3.3, \quad \chi_{\alpha}=0.5\right.$, $\left.r_{\alpha} / r_{1}=2.65\right)$ and $T_{e}(0)=10 \mathrm{keV}$, equation (11) reduces to $r_{\alpha} / a<0.6 k_{\| \alpha} / k_{\|}(r)$. This means that only waves with $k_{\| \alpha} / k_{\|}(r)>1$, which selects a certain sign of $m$, are weakly damped when $r_{\alpha} / a>0.6$. On the other hand, equation (12) is easily satisfied at given ratio $k_{\| \alpha} / k_{\| i}$ for $r_{1} \approx r_{\alpha}$, i.e. when the mode frequency is close to a harmonic of the ion gyrofrequency in the region where the instability source is located. However, $\left(r_{1}-r_{\alpha}\right)$ cannot be arbitrarily small because $k_{\| \alpha} \propto\left(r_{1}-r_{\alpha}\right)$, whereas we are considering the case of negligible drift, $k_{\| \alpha} v_{\| \alpha} \gg \omega_{D \alpha}$. This inequality yields:

$$
\frac{r_{\alpha}-r_{1}}{r_{\alpha}} \gg \frac{k_{\vartheta \alpha}}{k_{*}} \frac{v_{\| \alpha}}{v_{A *}} \frac{\rho_{\alpha}}{r_{\alpha}} \text {. }
$$

To obtain this equation, we assumed $\omega=k_{*} v_{A *}$ and used equation (7).

\subsection{The case of $k_{\| \alpha} v_{\| \alpha}<\omega_{D \alpha}$}

Neglecting longitudinal wavenumber in the resonance between alpha particles and the waves, we write the following resonance equation:

$$
\omega-l_{\alpha} \bar{\omega}_{B \alpha}\left(1-\frac{x_{\alpha}}{R}\right)=\omega_{D \alpha}(r, \vartheta) .
$$

The ratio $\omega_{D \alpha} / l \bar{\omega}_{B \alpha}$ is small. Therefore, it follows from (2) and (14) that $r_{1} \approx x_{\alpha}$ (i.e. $\omega$ is close to $l_{\alpha} \omega_{B \alpha}^{\min }$ for $\vartheta \ll 1$ ) when $r_{\alpha} / a \sim 1$.

For thermal ions we use equation (5). Eliminating $\omega$ from (5) and (14), we obtain:

$$
\frac{v_{\| i}^{\mathrm{res}}}{v_{T i}}=\frac{l_{\alpha} \bar{\omega}_{B i}}{k_{\| i} v_{T i}}\left[\frac{x_{i}-x_{\alpha}}{R}+\frac{j}{l_{\alpha}}\left(1-\frac{x_{i}}{R}\right)+\frac{\omega_{D \alpha}}{l \bar{\omega}_{B i}}\right] \text {. }
$$

Here $k_{\| i}$ is determined by (8), from which it follows that $k_{\| i}=m \Delta \iota / R$ in the considered case of very small $k_{\| \alpha}$. Then the factor before the brackets is very large, $l_{\alpha} \bar{\omega}_{B i} /\left(k_{\| i} v_{T i}\right) \sim R v_{A *} /\left(r v_{T i} \Delta \iota\right) \gg 1$. Therefore, the ion damping is very small, unless dominant terms in brackets compensate each other, i.e. 


$$
\frac{x_{\alpha}}{a} \approx \frac{x_{i}}{a}+A \frac{j}{l_{\alpha}},
$$

in which case

$$
\frac{v_{\| i}^{\mathrm{res}}}{v_{T i}}=\frac{\omega_{D \alpha}}{k_{\| i} v_{T i}} .
$$

Equation (16) shows that $x_{\alpha} \gg x_{i}$ when $l_{\alpha} \ll j R / r_{i}$. In particular, $x_{\alpha} / a$ lies in the range $0.5-0.9$ when $l_{\alpha}=4-9$ $\left(l_{i}=3-8\right) A=3.3 x_{i} / a=0.1, j=1$.

The ion resonance velocity given by equation (17) can be written as

$$
\frac{v_{\| i}^{\mathrm{res}}}{v_{T i}}=\frac{\rho_{\alpha}}{r_{\alpha}} \frac{v_{\alpha}}{v_{T i}} \frac{1+\chi_{\alpha}^{2}}{2 \Delta \iota} .
$$

Taking $\rho_{\alpha} / r_{\alpha}=0.1, v_{\alpha} / v_{T i}=10, \chi_{\alpha}=1 / 2$, and $2 \Delta \iota=1$, we obtain $v_{\| i}^{\text {res }} / v_{T i}=1.25$. This magnitude leads to $\gamma_{i} / \omega=$ $10^{-2}-10^{-4}$ for the modes with $l_{\alpha}=l_{i}+1=4-5$ (see appendix C). Thus, the ion damping can be favourable for the $\mathrm{SC}$ to the near-axis region.

The condition of small electron damping, $\omega>k_{\|}(r) v_{T e}$, is

$$
\frac{R v_{A_{*}} k_{*}}{r_{*} v_{T e}(r) \Delta \iota k_{\vartheta}}>1 \text {. }
$$

The inequality in (19) is well satisfied near the plasma edge due to low electron temperature. It can also be well satisfied in the core region. In order to see it, let us take again $\Delta \iota=0.5$, $R=300 \mathrm{~cm}$, and assume that $r_{*}=50 \mathrm{~cm}$ and $v_{T e} / v_{A *}=6$ (for instance, $T_{e}=10 \mathrm{keV}$ and $v_{A *}=10^{9} \mathrm{~cm} \mathrm{~s}^{-1}$ ), we obtain $\omega /\left|k_{\|}\right| v_{T e}=2 k_{*} /\left|k_{\vartheta}\right| \gg 1$. In this case the role of electron damping is small.

\section{Spatial energy transfer by FMM}

A necessary condition of the effective SC of the fast ion energy is that the waves should receive and radially transfer a large fraction of the energy of fast ions. Let us see whether this can be the case.

The power density received by the waves due to an instability driven by $\alpha$-particles is $P_{\alpha}^{w}=2 \gamma_{\alpha} W$, where $\gamma_{\alpha}$ is the fast ion drive (partial growth rate) and $W$ is the wave energy density. One can show that $W=\tilde{B}^{2} /(4 \pi)$ ( $\tilde{B}$ is the perturbed magnetic field) for FMM with $\omega^{2} \gg \omega_{B i}^{2}$ when the longitudinal wave number is sufficiently small, $k_{\|}^{2} / k_{\perp}^{2} \ll \omega_{B i}^{2} / \omega^{2}$. Taking this into account and assuming $\omega \approx l \omega_{B i}$, it is convenient to write $P_{\alpha}^{w}$ as follows:

$$
P_{\alpha}^{w}=2 l \omega_{B \alpha} \frac{\gamma_{\alpha}}{\omega} \frac{B^{2}}{4 \pi}\left(\frac{\tilde{B}}{B}\right)^{2}
$$

For instance, taking $B=3.5 \mathrm{~T}, l=5$ (which corresponds to $f_{w}=133 \mathrm{MHz}$ when $\omega_{B \alpha}$ is the gyrofrequency of alpha particles), $\gamma_{\alpha} / \omega=10^{-3}$, we obtain $P_{\alpha}^{w}=1.63 \times 10^{7}(\tilde{B} / B)^{2}$ $\mathrm{W} \mathrm{cm}^{-3}$. On the other hand, the fusion alpha power is $P_{\alpha}^{\mathrm{DT}}=\mathcal{E}_{\alpha} n_{\mathrm{D}} n_{\mathrm{T}}\langle\sigma v\rangle$, with $\mathcal{E}_{\alpha}=3.5 \mathrm{MeV}$. For $T_{i}=10 \mathrm{keV}$ (at $r / a \sim 1 / 2$ ) and $n_{i}=n_{\mathrm{D}}+n_{\mathrm{T}}=4 \times 10^{13} \mathrm{~cm}^{-3}, n_{\mathrm{D}}=n_{\mathrm{T}}$

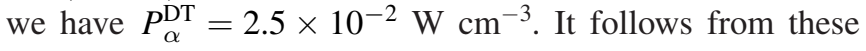

relations that rather low wave amplitudes are sufficient for the wave to extract from alphas the power density as large as the fusion produced power density. In the considered example, $P_{\alpha}^{w}=P_{\alpha}^{\mathrm{DT}}$ when $\tilde{B} / B=3.9 \times 10^{-5}$. Because in reality only a part of the alpha energy can be transferred to the waves, the required wave amplitudes are considerably less.

It is clear that the total power received by the waves is $\mathcal{P}_{\alpha}^{w}=\int_{V_{\text {ins }}} \mathrm{d}^{3} r P_{\alpha}^{w}$, where the integral is taken over the region where the instability is located, $V_{\text {ins }}$ is the volume of this region.

For the required SC the FMM should provide the energy transfer from the periphery region where the instability is located to the near-axis region. Therefore, below we consider this issue.

The eigenmodes in toroidal systems are actually waves standing in the radial direction. Therefore, an eigenmode can be treated as a superposition of two traveling waves, one of them moving outwards the plasma and another one moving inwards. In the absence of local sources and sinks (s\&s) the energy fluxes of the traveling waves in the steady state exactly compensate each other, so that the eigenmode energy flux $S^{w}=S_{+}+S_{-}=0$, where the subscripts ' + ' and '-' label the waves traveling outwards and inwards, respectively. The presence of local s\&s breaks the flux balance, leading to $S^{w} \neq 0$ and affecting the radial profile of the wave energy density. However, when s\&s are weak, the radial structure of the mode changes weakly. One can say that in this case the mode survives, although the energy is transferred from source regions to sink regions.

In order to obtain restrictions providing the persistence of the mode in the presence of s\&s, we have to write an equation for the mode energy density. Assuming that $S_{ \pm}= \pm\left|v_{g}^{t}\right| W_{ \pm}$we can write it as follows:

$$
\frac{\partial W}{\partial t}+\frac{1}{r} \frac{\partial}{\partial r} r S^{w}=2 \gamma^{L} W
$$

where $W=W_{+}+W_{-}, S^{w}=\left|v_{g}^{t}\right| \delta W, \delta W=W_{+}-W_{-}, v_{g}^{t}$ is the group velocity of the traveling waves that produce the standing wave, the term proportional to $\gamma$ describes local s\&s, $\gamma^{L}=\gamma_{\alpha}^{L}+\gamma_{d}^{L}$, with $\gamma_{\alpha}^{L}>0$ representing the local drive of the instability by the fast ions and $\gamma_{d}^{L}<0$ representing the mode local damping; the instability growth rate is given by $\gamma=\int \mathrm{d}^{3} r \gamma^{L} W / \int \mathrm{d}^{3} r W$ where the integral is taken over the plasma volume. Note that the energy flux in equation (21) can be written in the form of the flux for the traveling wave $\left(S^{w}=v_{g} W\right)$ with $v_{g}$ defined by

$$
v_{g} \equiv v_{g}^{t} \frac{\delta W}{W} .
$$

A steady state solution of equation (21) is $S^{w}=0$ (which implies that $\delta W=0$ ) in a particular case when the driving region coincides with the region where the mode is damped and $\gamma_{\alpha}(r)=-\gamma_{d}(r)$. Then the SC is absent. In general, however, $\delta W \neq 0$, even when the system is in steady state:

$$
S^{w}(r) \equiv\left|v_{g}^{t}\right| \delta W(r)=\frac{2}{r} \int_{0}^{r} \mathrm{~d} r_{1} r_{1} \gamma^{L}\left(r_{1}\right) W\left(r_{1}\right) .
$$


The integrand in this equation coincides with that in the numerator of $\gamma$, and $\gamma=0$ in the steady state. Therefore, the energy flux (23) vanishes when the region of integration includes the whole region of the mode location (or $r$ is less than radii where the mode is located, in which case $W=0$ in the integrand). On the other hand, $S^{w}(r) \neq 0$, inside the mode and depends of the distribution of sources and sinks. When sinks dominate at small radii, whereas sources dominate at larger radii, $S^{w}(r)<0$ within the mode location. This implies that the energy flux is directed inwards.

The mode survives, i.e. its radial shape $[W(r)]$ is approximately the same as in the absence of the instability, when $\delta W \ll W$. Using this inequality and equation (23), we can obtain a simple condition of eigenmode persistence for the case when the region where fast ions drive the instability does not overlap with the region of damping. Assuming that the alpha drive dominates in the region with the width $\Delta_{\alpha}$ and the wave damping dominates in the region with the width $\Delta_{d}$, we write the inequality $\delta W \ll W$ in the form:

$$
\gamma_{d} \Delta_{d}=\gamma_{\alpha} \Delta_{\alpha} \ll v_{g}^{t}
$$

When this condition is not satisfied, the eigenmode becomes an energetic-particle mode (EPM).

For $\omega=k v_{A}$, we can evaluate the wave group velocity as $v_{g}^{t}=k_{r} v_{A} / k=2 \pi v_{A} /\left(L_{m} k\right)$, with $k=\omega / v_{A}$. Then, taking $\omega=l_{\alpha} \omega_{B \alpha}$, we obtain from equation (24) the following restriction on the damping rate:

$$
\frac{\gamma_{d}}{\omega} \ll 2 \pi \frac{L_{m}}{\Delta_{d}}\left(\frac{v_{A}}{l_{\alpha} \omega_{B \alpha} L_{m}}\right)^{2} .
$$

As an example, let us take $l_{\alpha}=5, \Delta_{d} / L_{m}=1 / 4, L_{m}=40 \mathrm{~cm}$. Then we obtain that the eigenmode survives for $\gamma_{d} / \omega \ll 10^{-2}$.

It is of interest to see whether the energy flux of the mode $\left(S^{w}\right)$ can exceed the thermal flux of the bulk ion plasma $\left(S^{T}\right)$. For $S^{w}=v_{g} W$ and $S^{T}=\kappa_{\mathrm{T}} n_{i} T_{i} / L$, we obtain that $S^{w} \geqslant S^{T}$ provided that

$$
\frac{W}{n_{e} T} \geqslant \frac{\kappa_{\mathrm{T}}}{v_{g} L} .
$$

In the steady state $v_{g}=\gamma_{\alpha} \Delta_{\alpha}$, as follows from equation (23). Taking this into account and that $W=2(\tilde{B} / B)^{2} / \beta_{i}$, we can write equation (26) as follows:

$$
\frac{\tilde{B}}{B}>\left(\frac{\beta_{i}}{2} \frac{\omega}{\gamma_{\alpha}} \frac{\kappa_{\mathrm{T}}}{l_{\alpha} \omega_{B \alpha} \Delta_{\alpha} L_{m}}\right)^{1 / 2} .
$$

In particular, when $\beta_{i}=0.01, \kappa_{\mathrm{T}}=10^{4} \mathrm{~cm}^{2} \mathrm{~s}^{-1}, l=4$, $\gamma_{\alpha} / \omega=10^{-3}, \Delta_{\alpha}=10 \mathrm{~cm}, L_{m}=40 \mathrm{~cm}, B=3.5 \mathrm{~T}$, we obtain $\tilde{B} / B>4.2 \times 10^{-4}$.

\section{Inward spatial channelling in a one-fluid model}

In section 2 we have shown that the waves with frequencies close to harmonics of the ion gyrofrequency can simultaneously be in resonance with energetic ions at the plasma periphery and with thermal ions in the plasma core. However, the analysis carried out does not give an answer to the question whether this SC can improve the energy confinement time and increase the plasma temperature considerably. Below we consider this issue by employing a simple 1D model.

Assuming that the SC takes place, we proceed from the following steady state equation of the energy balance in a fully ignited reactor (i.e. reactor without auxiliary heating):

$$
\frac{1}{r} \frac{\mathrm{d}}{\mathrm{d} r} r \mathcal{K} \frac{\mathrm{d} T}{\mathrm{~d} r}+Q^{c}+Q^{w}=0,
$$

where $T$ is the plasma temperature (assume $T_{i}=T_{e}$ ), $\mathcal{K}=\kappa_{\mathrm{T}} n_{e}$ is the plasma thermal conduction coefficient, $Q^{c}$ describes the collisional plasma heating by alpha particles, $Q^{w}$ is the plasma heating by the waves which transfer the alpha energy to the plasma near-axis region, $\int \mathrm{d}^{3} r Q^{w}=\nu \mathcal{P}_{\alpha}$ and $\int \mathrm{d}^{3} r Q^{c}=(1-\nu) \mathcal{P}_{\alpha}$, the integral is taken over the plasma volume, $\mathcal{P}_{\alpha}$ is the total fusion power associated with alpha particles, $\nu$ is the fraction of this power received by the waves. The energy losses associated with the radiation are neglected.

A formal solution of equation (28) is

$$
T(r)=T(a)+\int_{r}^{a} \frac{\mathrm{d} r^{\prime}}{r^{\prime} \mathcal{K}} \int_{0}^{r^{\prime}} \mathrm{d} r^{\prime \prime} r^{\prime \prime}\left(Q^{c}+Q^{w}\right) .
$$

To calculate integrals in this equation, we assume that $Q^{w}=Q_{0}^{w} \exp \left(-\sigma_{w} r^{2} / a^{2}\right), Q^{c}=Q_{0}^{c} \exp \left(-\sigma_{c} r^{2} / a^{2}\right), \sigma_{w}$ and $\sigma_{c}$ are profile peaking factors, and $\mathcal{K}=$ const. One can see that $Q_{0}^{w}=\nu \sigma_{w} \mathcal{P}_{\alpha} /\left(J_{w} V_{p}\right)$ and $Q_{0}^{c}=(1-\nu) \sigma_{c} \mathcal{P}_{\alpha} /\left(J_{c} V_{p}\right)$, where $J_{j}=1-\exp \left(-\sigma_{j}\right)$ with $j=(c, w), V_{p}$ is the plasma volume. Taking this into account we obtain:

$$
T(r)=T(a)+\frac{a^{2} \mathcal{P}_{\alpha}}{4 \mathcal{K} V_{p}}\left[(1-\nu) \frac{I_{c}(\xi)}{J_{c}}+\nu \frac{I_{w}(\xi)}{J_{w}}\right],
$$

where $\quad \xi=r / a, \quad I(\xi)=\int_{\sigma_{j} \xi^{2}}^{\sigma_{j}}\left(1-\mathrm{e}^{-t}\right) t^{-1} \mathrm{~d} t . \quad$ At the magnetic axis $(r=0), I(\xi)$ reduces to the Ein function $\operatorname{Ein}(\sigma)=\int_{0}^{\sigma}\left(1-\mathrm{e}^{-t}\right) t^{-1} \mathrm{~d} t$, which can be approximated as $\operatorname{Ein}(\sigma) \approx \sigma$ for $\sigma \ll 1$ and $\operatorname{Ein}(\sigma) \approx \ln \sigma+0.577$ for $\sigma \geqslant 2$. In particular, when $\sigma_{c} \ll 1$, equation (30) reads:

$$
T_{0}=\frac{a^{2} \mathcal{P}_{\alpha}}{4 \mathcal{K} V_{p}}\left[1+\nu\left(\frac{I_{w}(0)}{J_{w}}-1\right)\right],
$$

where $T_{0}=T(0)$. Here the first term describes the temperature in the absence of SC. Hence, the SC plays a considerable role when the second term is not small. It follows from (31) that in the case of $\sigma_{w} \gg 1$ the SC doubles the plasma temperature in the near-axis region when

$$
\nu\left(\ln \sigma_{w}-0.42\right)=1 .
$$

For instance, equation (32) requires $\nu=0.53$ for $\sigma_{w}=10$. This satisfies the evident restriction of $\nu \leqslant 1$. The influence of the SC on the central temperature is demonstrated in figure 2.

Thus, a strong influence of the SC on the energy balance seems possible. The magnitudes of $\nu, Q^{c}(\xi)$ and $Q^{w}(\xi)$ depend on experimental conditions. Therefore, in order to know whether equation (32) is satisfied in a particular experiment, a dedicated analysis is required. 


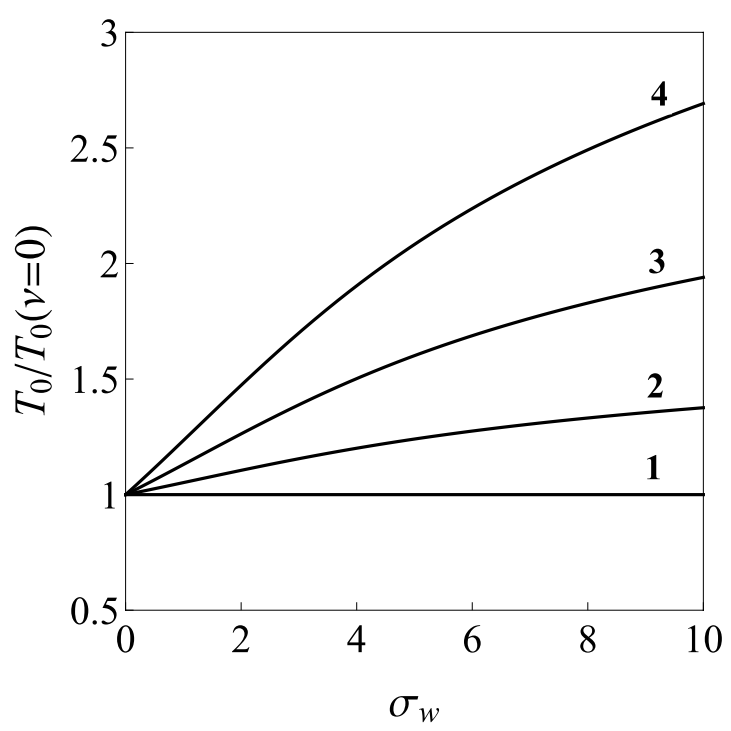

Figure 2. Dependence of the central plasma temperature in an ignited reactor on the profile peaking factor for the wave power deposition, $\sigma_{w}$, for various magnitudes of the fraction of the alpha energy $(\nu)$ transferred to the waves: $1, \nu=0 ; 2, \nu=0.2 ; 3$, $\nu=0.5 ; 4, \nu=0.9$.

\section{Conditions for a hot-ion mode}

The fusion power density is $P_{\text {fus }} \propto n_{i}^{2}\langle\sigma v\rangle \propto B^{2} \beta^{2}(1+$ $\left.T_{e} / T_{i}\right)^{-2}\langle\sigma v\rangle / T_{i}^{2}$. In the range of $10-20 \mathrm{keV},\langle\sigma v\rangle / T_{i}^{2} \approx$ const. Therefore, for given $\beta$ and the magnetic field, the fusion reaction rate is maximum when the ion temperature, $T_{i}$, exceeds the electron one, $T_{e}$. Therefore, it is of interest to find the conditions that should be satisfied to provide $T_{i}>T_{e}$.

We restrict ourselves to an analysis based on 0D equations for the energy balance of the ions and electrons. For a steady state plasma we can write:

$$
\begin{gathered}
\frac{3}{2} n_{i}\left(\frac{T_{i}}{\tau_{E i}}+\frac{\Delta T}{\tau_{e i}^{\mathcal{E}}}\right)=Q_{i}, \\
\frac{3}{2} n_{i}\left(\frac{T_{e}}{\tau_{E e}}\right)+Q_{\mathrm{br}}=\frac{3}{2} n_{i}\left(\frac{\Delta T}{\tau_{e i}^{\mathcal{E}}}\right)+Q_{e},
\end{gathered}
$$

where $\Delta T=T_{i}-T_{e} \geqslant 0, \tau_{E}$ is the energy confinement time, $\tau_{e i}^{\mathcal{E}}$ is the collisional time of the energy exchange between the plasma components (ions and electrons), $Q_{i}$ and $Q_{e}$ are the heating terms, $Q_{\mathrm{br}}$ describes bremsstrahlung, and $n_{e}=n_{i}$ was assumed. We assume that the fusion power well exceeds the power loss caused by bremsstrahlung. The LHS of equations (33) and (34) represent the loss of the energy, whereas the RHS are the energy gain. We can conclude that when $\Theta \equiv \tau_{e i}^{\mathcal{E}} / \tau_{E e} \ll 1$ and $\tau_{E e} \leqslant \tau_{E i}$, there is no solution with $\Delta T \sim T_{i}$ (i.e. $T_{i} \gg T_{e}$ ) of (33) and (34). To see it, we note that, according to (33), $n_{i} \Delta T \sim Q_{i} \tau_{e i}^{\mathcal{E}}$ for $\Delta T \sim T_{i}, \Theta \ll 1$; therefore, bremsstrahlung in equation (34) is negligible. Then it follows from (34) that the energy exchange between the plasma components is so strong that it makes $T_{e} \approx T_{i}$ even when $Q_{e}=0$. This implies that even an unrealistically strong energy channelling to the ions will lead to the ion temperature which only sightly exceeds the electron temperature. The situation changes when $\Theta$ is not small, then $T_{i} \gg T_{e}$ is possible. Thus, $\Theta$ is a key parameter which determines possible difference between $T_{e}$ and $T_{i}$. Because $\tau_{e i}^{\mathcal{E}} \propto T_{e}^{3 / 2} / n_{e}$, one can expect that a hot-ion regime can be achieved in experiments with high electron temperature and low plasma density. However, in a self-sustained fusion reactor there is a requirement $n_{i} \tau_{E}>10^{20} \mathrm{~m}^{-3} \mathrm{~s}$; therefore, when $\tau_{E e} \sim \tau_{E}, \Theta$ does not depend on the plasma density. In this case

$$
\Theta \lesssim 10^{-2} T_{e}^{3 / 2}
$$

where $T_{e}$ in $\mathrm{keV}$. We conclude that condition $\Theta \geqslant 1$, in which case $T_{i}$ can, in principle, exceed $T_{e}$ considerably due to the energy channelling, is satisfied for $T_{e}>20 \mathrm{keV}$.

\section{Effects of the SC in non-isothermic JET plasmas with NBI}

The foregoing indicates that the SC can, in principle, affect the plasma energy balance and provide considerable enlargement of the central temperature. However, the model used in section 4 does not show the difference between the temperatures of electrons and ions. Moreover, no specific JET parameters were included to the model. The presence of neutral beam injection was not taken into account.

In this section, our analysis will be carried within a more realistic model describing both $T_{i}$ and $T_{e}$, with prescribed other quantities (such as densities of electrons and ions, NBI heating, etc) taken from JET data base.

In order to observe effects of the SC, we will make calculations for three cases. First, for a pure deuterium plasma with prescribed plasma density and NBI heating. This will enable us to find thermal conductivity coefficients for the electrons and ions, which are required to provide the desired electron temperature and ion temperature. Second, using these conductivity coefficients we will make calculations for a DT plasma with the same NBI heating. Comparing the results of calculations for the first and second cases, we will observe effects of the alpha heating. The third case will differ from the second one only by adding the SC. The SC will be modeled as in section 4 , but with the additional assumption that the waves are damped due to the interaction with bulk plasma ions.

The following equations will be used:

$\frac{3}{2} n_{e} \frac{\partial T_{e}}{\partial t}-\frac{1}{r} \frac{\partial}{\partial r} r n_{e} \kappa_{e} \frac{\partial T_{e}}{\partial r}=Q_{e i}^{c}+Q_{\mathrm{NBI}}^{e}+Q_{e}^{c}-Q_{\mathrm{br}}$,

$\frac{3}{2} n_{i} \frac{\partial T_{i}}{\partial t}-\frac{1}{r} \frac{\partial}{\partial r} r n_{i} \kappa_{i} \frac{\partial T_{i}}{\partial r}=Q_{i e}^{c}+Q_{\mathrm{NBI}}^{i}+Q_{i}^{c}+Q^{w}$,

where the terms $Q_{e i}^{c}=-Q_{i e}^{c}$ describe the energy exchange between the electrons and ions through Coulomb collisions, $Q_{\mathrm{NBI}}^{i}$ and $Q_{\mathrm{NBI}}^{e}$ describe the NBI heating of the ions and electrons, respectively, $Q_{e}^{c}$ and $Q_{i}^{c}$ represent the collisional alpha heating of the electrons and ions, respectively, the subscript $e / i$ labels electrons / ions. As above, the term $Q^{w}$ describes the SC. These equations are written on the assumption that the temperatures of deuterium and tritium are equal. 
The terms $Q_{e i}^{c}$ and $Q_{\mathrm{br}}$ are well know. We take them in the form:

$$
\begin{gathered}
Q_{e i}^{c}=-\sum_{i=D, T} \frac{3 M_{e}}{M_{i}} \frac{n_{e}\left(T_{e}-T_{i}\right)}{\tau_{e}}, \\
Q_{\mathrm{br}}=\text { const } n_{e} n_{i} \sqrt{T_{e}},
\end{gathered}
$$

where $\tau_{e} \propto T_{e}^{3 / 2} / n_{i}$ is the electron-ion collision time.

The NBI heating terms, $Q_{\mathrm{NBI}}^{e}$ and $Q_{\mathrm{NBI}}^{i}$, will be obtained from TRANSP calculations in the JET data base.

As in section 4, we assume that the fraction of alpha power received by the waves is characterized by the $\nu$ parameter, $\int \mathrm{d}^{3} r Q^{w}(r)=\nu \mathcal{P}_{\alpha}$, and that the radial profile of the plasma heating by the waves is given by $Q^{w}(r)=Q_{0}^{w} \exp \left(-\sigma_{w} r^{2} / a^{2}\right)$, with $Q_{0}^{w}$ defined in section 4 . In addition, we assume that the waves are damped due to the interaction with the bulk plasma ions; therefore, the wave term is attributed to equation (37).

The collisional plasma heating by alpha particles will be calculated in two ways. First, by relying on the results of TRANSP calculations in JET database. Second, by using analytical expressions. The first way depends on particular shots. That is why it is of interest to test simple analytical formulas which clearly show dependence of $Q_{e}^{c}$ and $Q_{i}^{c}$ on plasma parameters.

(i) Let us describe first a way based on TRANSP calculations.

We proceed from the following obvious relationship: $(1-\nu) \mathcal{P}_{\alpha}=\int \mathrm{d}^{3} r\left[Q_{e}^{c}+Q_{i}^{c}\right]$, which can be written as

$$
(1-\nu) \mathcal{P}_{\alpha}=2 V_{p} Q_{e 0}^{c} \int_{0}^{1} \mathrm{~d} \xi \xi\left[\widetilde{Q}_{i}^{c}(\xi) q_{i e}+\widetilde{Q}_{e}^{c}(\xi)\right],
$$

where $\widetilde{Q}_{e}^{c}(\xi)=Q_{e}^{c} / Q_{e 0}^{c}, \widetilde{Q}_{i}^{c}(\xi)=Q_{i}^{c} / Q_{i 0}^{c}, q_{i e}=Q_{i 0} / Q_{e 0}$, and $Q_{0}^{c}=Q^{c}(0)$. This equation will enable us to find $Q_{e 0}^{c}$ provided that $q_{i e}$ and the profile functions $\widetilde{Q}_{e}^{c}(\xi), \widetilde{Q}_{i}^{c}(\xi)$ are known. The former is determined by the following equation:

$$
q_{i e}=\delta_{i e} \frac{\int_{0}^{1} \mathrm{~d} \xi \xi \widetilde{Q}_{e}^{c}(\xi)}{\int_{0}^{1} \mathrm{~d} \xi \xi \widetilde{Q}_{i}^{c}(\xi)},
$$

where $\delta_{i e}$ is the ratio of the total fusion energy received by ions to that received by electrons due to collisions, it describes collisional distribution of the alpha energy between electron and ions.

Equations (36)-(41) will be solved with neglecting the influence of the SC on $q_{i e}, \widetilde{Q}_{e}^{c}(\xi), \widetilde{Q}_{i}^{c}(\xi)$, and $\delta_{i e}$. These quantities and some others will be taken from TRANSP calculations in the absence of SC.

(ii) Equations for $Q_{i}^{c}$ and $Q_{e}^{c}$ were derived in [28] for a plasma with one ion species. They can be easily generalized for a DT plasma. As a result, we have:

$$
\begin{gathered}
Q_{i}^{c}=(1-\nu) S_{\alpha} \mathcal{E}_{\alpha} \zeta_{\alpha i}, \\
Q_{e}^{c}=(1-\nu) S_{\alpha} \mathcal{E}_{\alpha}\left(1-\zeta_{\alpha i}\right),
\end{gathered}
$$

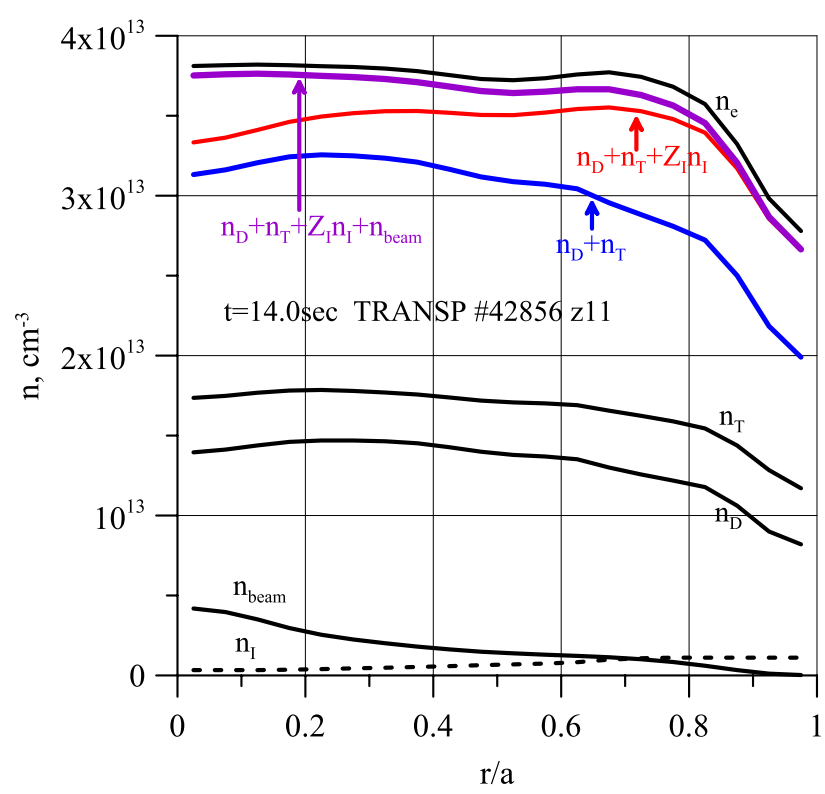

Figure 3. Profiles of electron density, thermal ion densities (deuterium, tritium and carbon), beam ions and total ion density $\left(n_{\mathrm{D}}+n_{\mathrm{T}}+Z_{I} n_{I}+n_{\text {beam }}\right)$ in discharge $\# 42856, t=54 \mathrm{~s}$.

where $S_{\alpha}$ is the alpha source function (both its thermonuclear part, $n_{\mathrm{D}} n_{\mathrm{T}}\langle\sigma v\rangle$, and the beam-plasma contribution are important), $\mathcal{E}_{\alpha}=3.5 \mathrm{MeV}$, and [28]

$$
\begin{gathered}
\zeta_{\alpha i}=\frac{2}{3} \eta^{2}\left[\sqrt{3}\left(\operatorname{arctg} \frac{2-\eta}{\sqrt{3} \eta}+\frac{\pi}{6}\right)-\frac{1}{2} \ln \frac{(1+\eta)^{3}}{1+\eta^{3}}\right], \\
\eta=\left[\frac{3 \sqrt{\pi}}{8} \frac{M_{\alpha}}{M_{\mathrm{DT}}}\left(\frac{M_{\alpha}}{M_{e}}\right)^{1 / 2}\right]^{1 / 3}\left(\frac{T_{e}}{\mathcal{E}_{\alpha}}\right)^{1 / 2} .
\end{gathered}
$$

$M_{\mathrm{DT}}=M_{\mathrm{D}} M_{\mathrm{T}} /\left(M_{\mathrm{D}}+M_{\mathrm{T}}\right), M$ is the particle mass.

Using these equations, we analyzed the effect of the SC as follows.

We selected discharges \#42856 and \#42847 with DT plasmas, see table A1. Taking into account that the electron densities in these discharges and deuterium discharge \#41069 were rather similar, with the intermediate magnitude in discharge \#42856, we used the particle density of discharge \#42856 in our calculations.

To begin with, we found $\kappa_{e}$ and $\kappa_{i}$ required to get the central electron temperature and the ion temperature close to those observed in the deuterium discharge \#41069. This was done by solving equations (36) and (37) with prescribed particle densities $n_{i}(r)$ and $n_{e}(r), Q_{\mathrm{NBI}}^{i}$ and $Q_{\mathrm{NBI}}^{e}$, which were taken from JET data base and shown in figures 3 and 4 . In calculations we took $\kappa_{i}(r)=$ const and $\kappa_{e}(r)=$ const. It was found that a satisfactory agreement with the experimental data (shown in table A1 in appendix B) is reached for $\kappa_{i}=5000 \mathrm{~cm}^{2} \mathrm{~s}^{-1}$ and $\kappa_{e}=2700 \mathrm{~cm}^{2} \mathrm{~s}^{-1}$, which yielded $T_{i 0}=13.35 \mathrm{keV}$ and $T_{e 0}=9.90 \mathrm{keV}$. These transport coefficients are actually some averages of the coefficients calculated by TRANSP. The modelling thermal conductivity by $\kappa_{e / i}(r)=$ const was made not only for simplicity. This choice enabled 


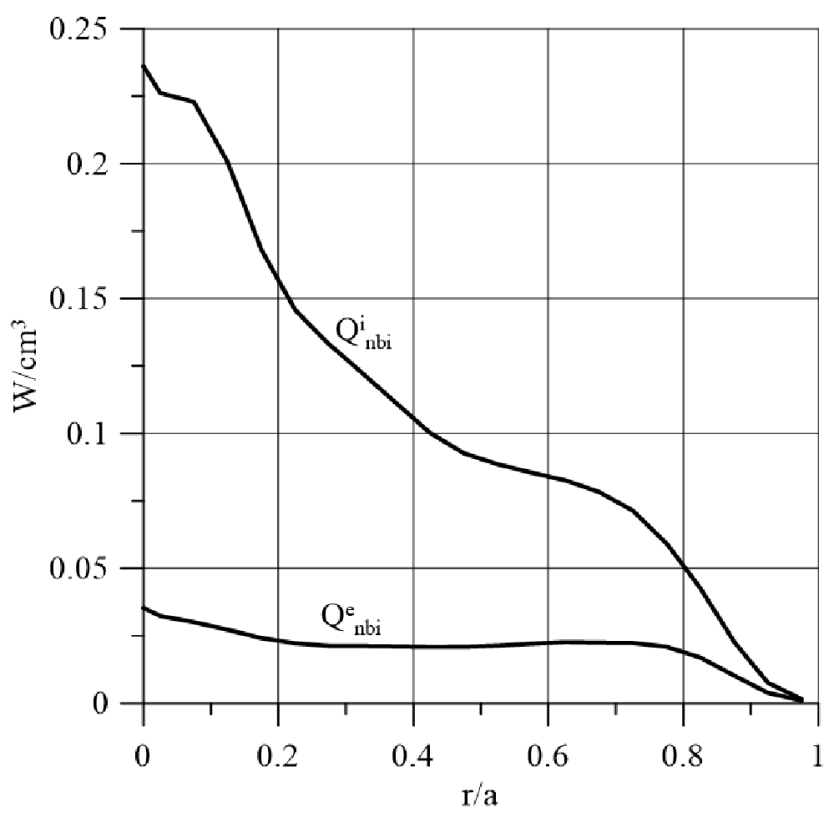

Figure 4. Sources $Q_{\mathrm{NBI}}^{i}$ and $Q_{\mathrm{NBI}}^{e}$ for the shot $\# 42856, t=54 \mathrm{~s}$.

us to single out effects of the SC. We mean that plasma transport coefficients, first of all, their radial structure, in DT plasmas may differ from those in deuterium plasma because of isotope effects, alpha heating, dependence of the threshold of the ion temperature gradient (ITG) instability on $T_{i} / T_{e}$, and the SC. In the presence of the $\mathrm{SC}$, it is meaningless to use transport coefficients $\kappa_{e / i}(r)$ calculated by TRANSP.

Therefore, our next step was to proceed to calculations for a DT plasma with the same $\kappa_{i}(r)=$ const and $\kappa_{e}(r)=$ const and different $\nu$. The results are shown in figure 5 and table 1. Comparing the first and second rows in table 1 we see that proceeding from deuterium plasma to a DT plasma with unchanged transport coefficients decreases the difference $T_{i}-T_{e}$ by increasing mainly the electron temperature. This is a natural result because the alpha-electron collisional time is less than the alpha-ion collisional time. The presence of the SC changes the situation, as seen from the third and fourth lines in table 1: The ion temperature increases but the electron temperature decreases; the SC with $\nu=0.2$ is sufficient to provide a noticeable effect. This effect grows approximately linearly, as follows from figure 5 .

We remind that the alpha power in the considered experiments was much less than the NBI power, being about $15 \%$ of $\mathcal{P}_{\mathrm{NBI}}$. This implies that the SC will have much stronger influence on the central temperatures of ions and electrons and cause stronger improvement of the plasma confinement in experiments with larger ratio $\mathcal{P}_{\alpha} / \mathcal{P}_{\mathrm{NBI}}$. On the other hand, it is clear that the predominant ion heating will not necessary take place because the electron damping can be considerable, see appendix $\mathrm{C}$.

The calculations with the use of $Q_{e}^{c}$ and $Q_{i}^{c}$ determined by equations (40) and (41) (which are relied on TRANSP) resulted in approximately the same results. This indicates that the approximation of these magnitudes by simple analytical expressions (42) and (43) is justified.

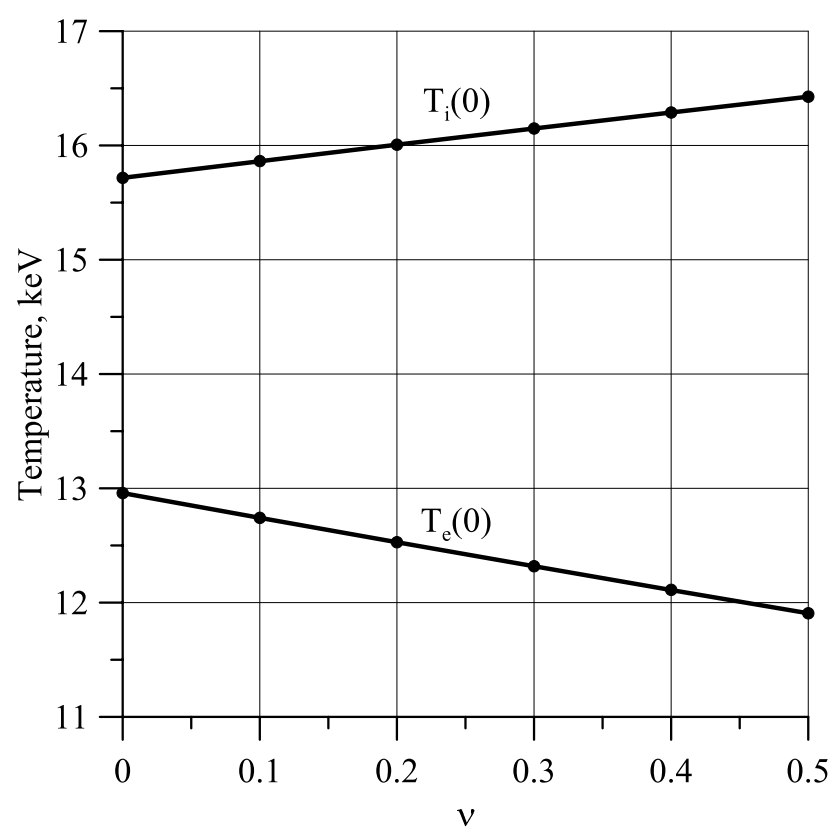

Figure 5. Dependence of the central ion and electron temperatures on the fraction of alpha energy $(\nu)$ transferred to the waves, which was obtained by solving the system of equations (36) and (37) for $\chi_{i}=5000 \mathrm{~cm}^{2} \mathrm{~s}^{-1}, \chi_{e}=2700 \mathrm{~cm}^{2} \mathrm{~s}^{-1}, \sigma_{w}=10$, and $Q_{i}^{c}, Q_{e}^{c}$ in the form of (42) and (43).

Table 1. Calculated steady-state central temperatures of the ions and electrons in the absence of the SC (two first rows) and in the presence of the SC (two last rows) during NBI injection with $\mathcal{P}=10 \mathrm{MW}$. The first row reproduces the temperatures in deuterium discharge \#41069. Two last rows demonstrate possible effects of the SC in DT discharges \#42856 and \#42847. In calculations we took $\chi_{i}=5000 \mathrm{~cm}^{2} \mathrm{~s}^{-1}, \chi_{e}=2700 \mathrm{~cm}^{2} \mathrm{~s}^{-1}$, $\sigma_{w}=10$, and the particle densities and NBI heating shown in figures 3 and 4 . These discharges were described in [1-3], see also our analysis in appendix A.

\begin{tabular}{llrl}
\hline Heating & $T_{i 0}, \mathrm{keV}$ & $T_{e 0}, \mathrm{keV}$ & $T_{i 0}-T_{e 0}$ \\
\hline $\mathrm{NBI}$ & 13.35 & 9.90 & 3.45 \\
$\mathrm{NBI}+\alpha$ & 15.71 & 12.96 & 2.75 \\
$\mathrm{NBI}+\alpha+\mathrm{SC}(\nu=0.2)$ & 16.01 & 12.53 & 3.48 \\
$\mathrm{NBI}+\alpha+\mathrm{SC}(\nu=0.3)$ & 16.15 & 12.32 & 3.83 \\
\hline
\end{tabular}

According to [1-3] and table A1 in appendix A, alpha heating in JET results in the increase of both the electron temperature and ion temperature. Our calculations in the absence and in the presence of the SC agree with this fact $T_{i 0}=13.35 \mathrm{keV}$ and $T_{e 0}=9.9 \mathrm{keV}$ in the first row of table 1 are less than the ion and electron temperatures shown in other rows of this table and in figure 5. However, comparing the results shown in tables 1 and A1 we observe that in the absence of the $\mathrm{SC}(\nu=0)$ the ion heating is underestimated but the electron heating is overestimated in our calculations based on the assumption that transport coefficients are the same in deuterium plasma and deuterium-tritium plasma. This overheating of electrons cannot be avoided without introducing additional degradation of the electron energy confinement time in DT plasmas, whereas better confinement of the ion energy is required to increase the ion temperature when $\nu=0$. The SC in DT plasmas increases 
the difference $\left(T_{i}-T_{e}\right)$ by providing an additional heating of the ions (which increases $T_{i}$ ) and decreasing the electron heating (which decreases $T_{e}$ ). Nevertheless, our calculations with $\nu=0.2$ and $\nu=0.3$ still lead to smaller $T_{i}$ and larger $T_{e}$ than those observed in the experiments. Increasing $\nu$, we obtain a better agreement with experiment $\left(T_{i}>16.2 \mathrm{keV}\right.$ and $T_{e} \lesssim 12 \mathrm{keV}$ ), as follows from figure 5 . However, it seems that $\nu>0.3$ is not realistic: calculations in straight magnetic field predict that $15-20 \%$ of the energy stored in $\alpha$-particles can be transferred to FMM [29]; a larger value $(50 \%)$ was obtained in [30], but it was actually an estimate made for another type of waves (GAE) with rather large amplitudes; to make a reliable prediction of the magnitude of $\nu$ for JET, a special research is required, which is beyond the scope of this work. In addition, some fraction of the FMM energy is to be absorbed by electrons, which was ignored in our calculations. Therefore, it seems probable that other factors, not only the SC, may contribute to the increase of $\left(T_{i}-T_{e}\right)$, in particular, ion transport reduction due to ITG turbulence stabilization [31-33]. We also wish to point out that the interpretation of the experiments was hampered by the non-stationary nature of the plasma discharges [34] (this issue is discussed in appendix A).

A more definite conclusion on the role of different physical mechanisms, in particular, the SC, in the improvement of the plasma performance should rely on a quantitative consideration. At present, this is not possible because the available theory and experimental database are not sufficient.

\section{Summary and conclusions}

Our analysis suggests that the SC may have played a role in the improved confinement and increased ion temperature in JET experiments with alpha particle heating. This conclusion is supported by the following:

(i) The fraction of the $3.5 \mathrm{MeV}$ alpha particles born in the peripheral region, $r / a \geqslant 0.5$, is considerable. In the discharge \#42856 this fraction is $\sim 30 \%$. Due to orbital motion, the fraction of alphas in the periphery increases. In particular, the fraction of trapped alphas having $r_{\max }$ at $r / a \geqslant 0.5$ reaches $50 \%$ for $\lambda=1$ in the mentioned discharge.

(ii) A necessary condition for the $\mathrm{SC}$ - the resonance interaction between FMM destabilized in the peripheral region and the bulk plasma in the near-axis region $\left(r_{i} \ll r_{\alpha}\right)$ can be satisfied for FMM with the frequencies close to cyclotron harmonics of alpha particles.

(iii) The wave damping rate in the plasma central region can be in the range $\gamma_{d}=\left(10^{-2}-10^{-4}\right) \omega$. This is comparable to the magnitude of the instability growth rate expected due to velocity anisotropy of the distribution function of peripheral alpha particles. Note that the ICE observed in JET and other tokamaks was associated with peripheral FMM instabilities having $\gamma_{\alpha} / \omega$ in this range.

(iv) The wave damping in the near-axis region is associated with both ions and electrons. This means that the SC can deliver the alpha energy not only to bulk plasma ions but also to electrons. Conditions providing dominant ion damping are found.

(v) Reasonably small amplitude waves can receive and transfer across the flux surfaces as large power density as that required for spatial channelling of a considerable part of fusion energy.

(vi) There is a qualitative agreement between the experimentally measured electron and ion temperatures in JET DT discharges and the temperatures calculated numerically with the assumptions that, first, transport coefficients in DT plasmas are the same as those in a deuterium discharge and, second, the inward SC leading to the heating of the ions in the plasma core takes place in DT discharges.

When points (i)-(iii) are true, the plasma energy confinement time increases. When the ion damping dominates over the electron one, the SC leads mainly to the increase of the ion temperature in the plasma core.

An analysis of JET experimental data was carried out, which confirmed the conclusion of [1-4] that the energy content in DT discharges exceeds that in deuterium discharges with alpha power substituted by ICRH power. In addition, it was shown that the plasma energy confinement time in the considered ICRH discharges is minimum at the highest ICRH power.

A number of issues are to be clarified in order to make a reliable conclusion on the role of the SC in JET. First of all, experimental and theoretical studies are required to investigate the existence of FMM with a considerable radial extent, as well as the destabilization of multiple FMM by alpha particles and other energetic ions. At present, to our knowledge, the radial structure of FMM was measured only in NSTX experiments, which supports our assumption on the mode structure. However, in NSTX the FMM were driven by NBI with the beam particle energies well below the fusion alpha energy and the wave frequencies were below the ion gyrofrequency.

A necessary condition of the efficient SC is transferring of a large fraction of the fast-ion energy to the waves, i.e. anomalous slowing down of fast ions. This issue was considered in [30] for GAE modes. It was found that five GAE modes with the frequencies of $\sim \omega_{B i} / 3$ and the amplitudes about $\tilde{B}_{r} / B=5 \times 10^{-3}$ are sufficient to make the motion of injected ions in NSTX stochastic in the energy range from $50 \mathrm{keV}$ to $90 \mathrm{keV}$. This meant that these GAEs are expected to extract about a half of the energy of the $90 \mathrm{keV}$ ions driving the instability. The fraction of the energy transfer from energetic ions to FMM with $\omega>\omega_{B i}$ is to be studied.

We should also not forget that the improved confinement in the JET DT plasmas may be due to other effects, such as ITG stabilization by fast ions [32] and to a favourable scaling of confinement with ion mass, as seen in JET $[35,36]$ and JT-60U [37]. Additionally, the interpretation of these discharges is difficult due to their transient nature and due to changes in sawtooth behavior with the isotope mass [34]. To conclude, our analysis shows that the SC of alpha particle power can play an important role in fusion plasmas. Although it may not be possible to ascertain that the SC played a role in the JET 
experiments described here, the inward SC has the potential of significantly enhancing the performance of a fusion reactor, by channeling alpha power from the periphery to the core.

\section{Acknowledgments}

The authors thank L. Garzotti, V. Kiptily, and K.G. McClements for their comments made during presentation of this work at the JET seminar, October 18, 2017.

This work was carried out within the framework of the EUROfusion Consortium and received funding from the EURATOM research and training programme 2014-2018 under grant agreement No. 633053. The views and opinions expressed herein do not necessarily reflect those of the European Commission. The work was also supported by the STCU Project No. 6392 and NASU Project No. PL15/17.

\section{Appendix A. Experimental data base}

Analysis of experimental data obtained during the DTE1 campaign on JET indicates the improvement of the plasma confinement time in discharges with DT plasmas in comparison to those with deuterium plasmas where the plasma heating by alpha particles was substituted with ICRH heating. Figure A1 employing the parameters given in table A1 demonstrates this. It shows that maximum $W_{\text {dia }}$ in a DT plasma with $P_{\alpha}=1.3-1.4 \mathrm{MW}$ exceeds that in deuterium plasma with the ICRH heating power in the range $(0-2.1) \mathrm{MW}$ by a factor of $\sim 1.6$. All the considered discharges contained fast ions produced by NBI. In addition, DT discharges contained fusion produced alpha particles, and deuterium discharges \#41067 and \#41068 contained ions accelerated by ICRH. This implies that thermal energy in both DT and D discharges was less than the magnitudes shown in figure A1. However, the magnitude of the $W_{\text {dia }}^{\mathrm{DT}} / W_{\text {dia }}^{D}$ ratio for thermal plasma is hardly less considerably than that determined by figure A1. Note that $\left[n_{e 0}\left(T_{i 0}+T_{e 0}\right)\right]^{\mathrm{DT}} /\left[n_{e 0}\left(T_{i 0}+T_{e 0}\right)\right]^{\mathrm{ICRH}} \approx 1.3$, where the subscript ' 0 ' labels magnitudes at the magnetic axis. This ratio is in a qualitative agreement with the magnitude of $W_{\mathrm{dia}}^{\mathrm{DT}} / W_{\mathrm{dia}}^{D}$.

It also follows from figure A1 that the plasma heating by ICRH with up to $P_{\mathrm{ICRH}}=2.1 \mathrm{MW}$ only slightly increases $W_{\text {dia. }}$. This implies that the ICRH leads to the degradation of the plasma confinement time $\left(\tau_{E}\right)$, which confirms the known fact that the auxiliary heating tends to deteriorate the plasma energy confinement time. To evaluate the rate of degradation of $\tau_{E}$, we have to take into account that the NBI with $P_{\mathrm{NBI}}=10 \mathrm{MW}$ took place in all the considered discharges, which means that the ICRH increased the heating power by $\leqslant 21 \%$, while $W_{\text {dia }}$ increased by $\lesssim 10 \%$ only (as follows from figure A1). One can see that the degradation of $\tau_{E}$ agrees with the law $\tau_{E} \propto 1 / \sqrt{P_{\text {total }}}: \tau_{E} / \tau_{E 0}=\sqrt{P_{\mathrm{NBI}} / P_{\mathrm{NBI}}+\mathrm{ICRH}}=0.9$.

Other observations documented in table A1: First, the ion temperature always exceeds the electron one, which was expected because both NBI and ICRH heated mainly ions. Second, substituting the ICRH power with the fusion alpha power increases the difference between the central ion temperature and electron temperature $\left(T_{i 0}-T_{e 0}\right)$. This means that, in addition to Coulomb collisions leading to preferable electron heating by alphas, there exists another heating mechanism which, in contrast to Coulomb collisions, heats the ions. Moreover, this mechanism is more powerful, as follows from the fact that the ion temperature in DT plasmas exceeds the temperature in deuterium discharges with ICRH.

Note that in the considered discharges sawtooth oscillations took place. Therefore, a question arises whether they affect the conclusions drawn. Below we discuss relevant issues.

(i) Time between sawtooth crashes $\left(\tau_{\text {saw }}\right)$ is quite considerable, $\tau_{\text {saw }}=1-1.5 \mathrm{~s}$, which exceeds the crash duration by several orders. The maximum temperature takes place before the crash. Just these magnitudes are shown in table 1 .

(ii) $\tau_{\text {saw }}$ before maximum of $T_{i 0}$ is reached in DT discharges is smaller than that in deuterium discharges. Therefore, one can expect that the effect of the ion heating in DT plasmas would be even stronger if crashes were absent.

(iii) Collisional slowing down time of alphas is $\tau_{\alpha}^{\mathcal{E}} \sim 0.6 \mathrm{~s}$ $\left[(0.4-0.8) \mathrm{s}\right.$ for $\left.T_{e}=(10-15) \mathrm{keV}, n_{e}=4 \times 10^{19} \mathrm{~m}^{-3}\right]$. This time is less than $\tau_{\text {saw }}$, but not negligible. Therefore, effects of finite $\tau_{\alpha}^{\mathcal{E}}$ on the plasma heating can be noticeable. However, the orbit width of near-axis alphas is comparable to the $q=1$ radius, which minimizes the influence of sawteeth on alpha heating.

(iv) The sawtooth mixing radius in the considered discharges can be evaluated as $1 / 4-1 / 3$ of the plasma radius, so that only $\sim 1 / 10$ part of the plasma volume is affected. As plasma pressure profiles are decreasing (mainly, due to the temperature profiles because the particle density profiles are flat or even slightly hollow), the fraction of the plasma energy in this region is higher, but it hardly can considerably exceed $10 \%$. The sawtooth crashes change the plasma central temperature by $30 \%$ and, therefore, not more than $3 \%$ of the plasma energy is redistributed. For this reason, the influence of sawtooth crashes on the ratio $W_{\text {dia }}^{\mathrm{DT}} / W_{\text {dia }}^{D}$ for thermal particles is rather small.

\section{Appendix B. Fraction of alpha particles with the birth energy at the plasma periphery}

The SC providing the transfer of the alpha energy from the periphery to the near-axis region can be efficient provided that the number of alpha particles with the energy about $3.5 \mathrm{MeV}$ is considerable at the periphery. Therefore, we have to evaluate the number of alphas in this region. First of all, let us consider the radial dependence of the rate of the production of alpha particles due to both thermonuclear reaction and beam-plasma reaction, $S_{\alpha}(r)$. This magnitude and the function $r S_{\alpha}(r)$ are shown for discharges \#42856 and \#42847 in figure B1. The function $r S_{\alpha}(r)$ enables us to see directly the rate of production of $3.5 \mathrm{MeV}$ alpha particles on each flux surface. We observe that a maximum of $r S_{\alpha}(r)$ is located close to the flux surface $r / a \approx 0.3$ in the discharge \#42856 and $r / a \approx 0.4$ in the discharge \#42847, most of alphas are born in 
Table A1. Plasma parameters in deuterium discharges and deuterium-tritium discharges at the moments of time when temperatures of ions and electrons were maximum (before a sawtooth crash), see [1,2].

\begin{tabular}{|c|c|c|c|c|c|c|}
\hline Discharge & Heating & $T_{i 0}, \mathrm{keV}$ & $T_{e 0}, \mathrm{keV}$ & $T_{i 0}-T_{e 0}$ & $n_{e 0}, \mathrm{~m}^{-3}$ & $t, \mathrm{~s}$ \\
\hline \#41069 Deuterium & 10 MW NBI & 13.3 & 9.9 & 3.4 & $3.8 \times 10^{19}$ & 53.5 \\
\hline \#41067 Deuterium & $10 \mathrm{MW}$ NBI $0.9 \mathrm{MW}$ ICRH & 14.5 & 10.4 & 4.1 & $3.6 \times 10^{19}$ & 53.5 \\
\hline \#41068 Deuterium & $10 \mathrm{MW}$ NBI $2.0 \mathrm{MW}$ ICRH & 14.5 & 10.3 & 4.2 & $3.6 \times 10^{19}$ & 53.5 \\
\hline \#42856 Deuterium $(50 \%)+$ tritium $(50 \%)$ & $10 \mathrm{MW}$ NBI $1.34 \mathrm{MW} P_{\alpha}$ & 16.5 & 11.5 & 5.0 & $3.9 \times 10^{19}$ & 54.0 \\
\hline \#42847 Deuterium $(25 \%)+$ tritium $(75 \%)$ & 10 MW NBI 1.4 MW $P_{\alpha}$ & 17.5 & 12.0 & 5.5 & $4.1 \times 10^{19}$ & 54.0 \\
\hline
\end{tabular}

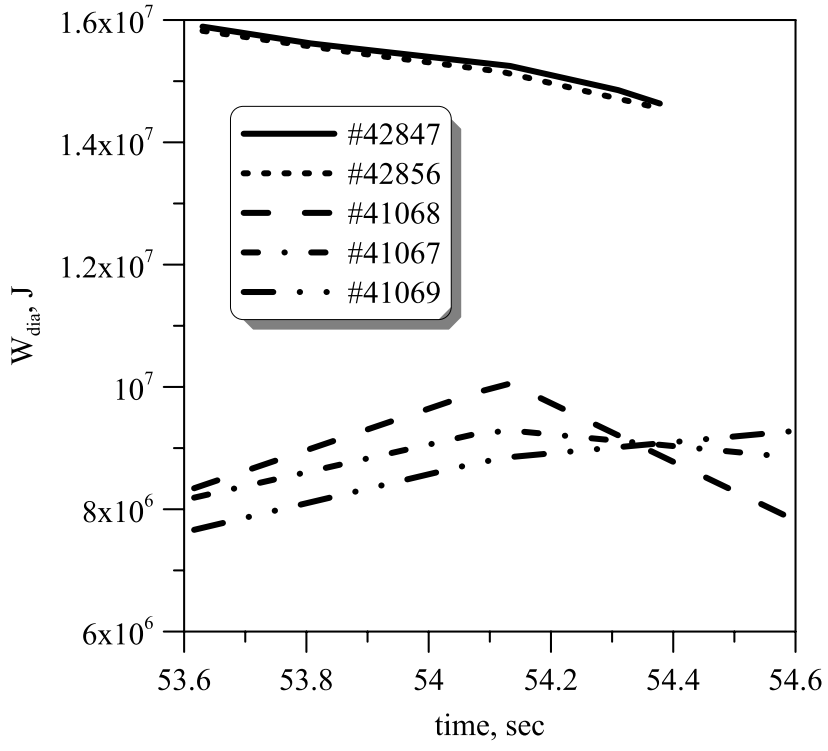

Figure A1. Measured diamagnetic energy in three discharges with $D D$-plasmas (\#41069, \#41067, \#41068) and two discharges with DT-plasma (\#42847, \#42856): the upper curves, discharge $\# 42847$ (50\% D and 50\% T) with $P_{\alpha}=1.4 \mathrm{MW}, P_{\mathrm{ICRH}}=0$ and discharge \#42856 (50\% D and 50\% T) with $P_{\alpha}=1.34 \mathrm{MW}$, $P_{\text {ICRH }}=0$; the lowest curve, discharge \#41069 with $P_{\text {ICRH }}=0$; two curves above the lowest one correspond to discharges \#41067 with $P_{\mathrm{ICRH}}=0.9 \mathrm{MW}$ and $\# 41068$ with $P_{\mathrm{ICRH}}=2.5 \mathrm{MW}$. In all these discharges $P_{\mathrm{NBI}}=10 \mathrm{MW}$.

the region $0.1<r / a<0.6$. So, the number of alpha particles born at $r / a>1 / 2$ is considerable.

For a more definite conclusion we have to calculate the function $\mathcal{F}(r)$ describing the fraction of alpha particles with $\mathcal{E}_{\alpha}=3.5 \mathrm{MeV}$ born in the region outside a certain radius $r$. This function is given by

$$
\mathcal{F}^{(0)}(r) \equiv \frac{\int_{r}^{a} \mathrm{~d} r_{0} r_{0} S_{\alpha}\left(r_{0}\right)}{\int_{0}^{a} \mathrm{~d} r_{0} r_{0} S_{\alpha}\left(r_{0}\right)},
$$

where $r<a$, the superscript ' 0 ' means that no orbit effects are included, the integrand contains alpha birth profile. Calculating integrals in (B.1), we obtain the result shown in figure B2. It follows from this figure that about $30 \%$ of alphas are born outside the flux surface with $r / a \geqslant 0.5$.

After the birth, alpha particles move along various orbits. During this motion, they deflect outwards and inwards from flux surfaces. Because $S_{\alpha}(r)$ is a monotonic decreasing function, the main effect of the orbital motion on the $3.5 \mathrm{MeV}$ alpha density is the broadening of its radial profile. This implies that

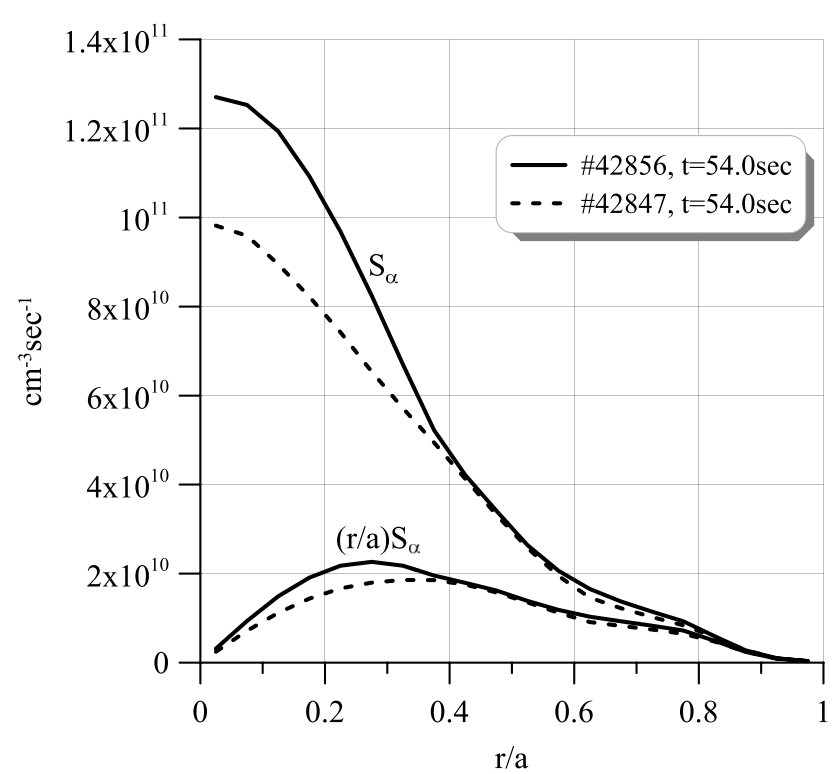

Figure B1. Fusion reaction rate, $S_{\alpha}(r)$, versus $r$ and its distribution over flux surfaces, $r S_{\alpha}(r)$, in JET discharges \#42847 and \#42856.

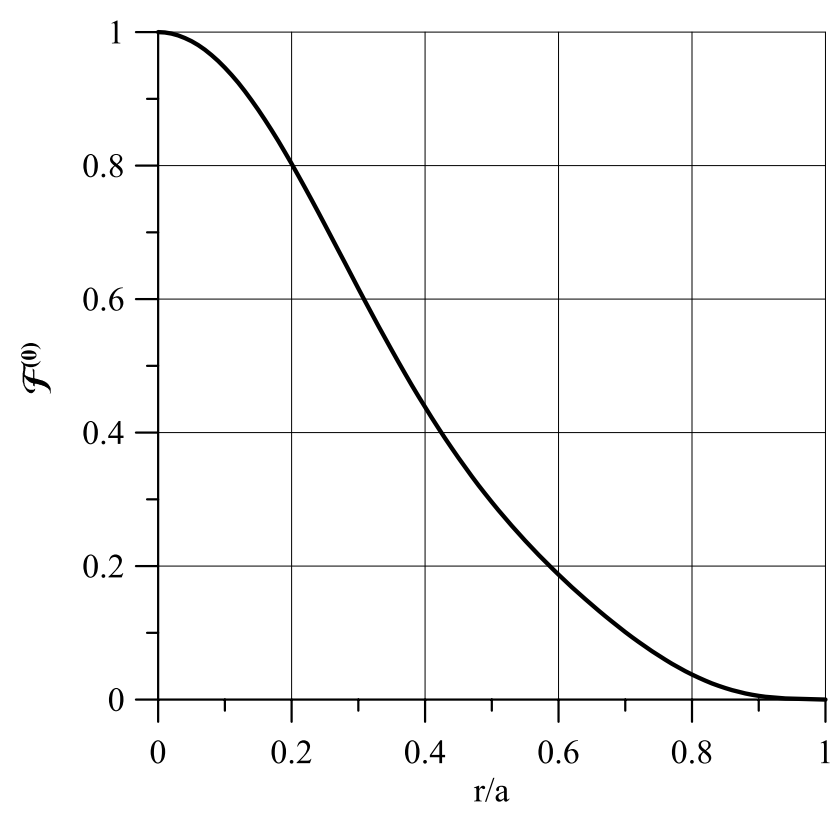

Figure B2. Fraction of alpha particles with the birth energy outside a certain flux surface with the radius $r$ in the discharge \#42856, calculated neglecting the orbit width.

the number of peripheral alphas increases, although some of them are lost to the wall. One more factor increasing the number of peripheral $3.5 \mathrm{MeV}$ alpha particles is the stochastic 
(collisionless and weakly collisional) diffusion, see, e.g. [38, 39]. Below we consider the profile broadening by taking into account the orbital motion only.

Because the alpha particle transit/bounce time $\left(\tau_{\mathrm{b}}\right)$ is much less than the slowing down time $\left(\tau_{\mathrm{s}}\right)$, we can introduce the bounce/transit averaged distribution function of the population of the alphas with $\mathcal{E} \sim 3.5 \mathrm{MeV}$ :

$$
F_{\alpha}=\frac{\Delta t}{4 \pi v_{\alpha}^{2}} \delta\left(v-v_{\alpha}\right)\left\langle S_{\alpha}\right\rangle
$$

where $\left\langle S_{\alpha}\right\rangle=\oint \mathrm{d} t S_{\alpha}[r(t)] / \tau_{\mathrm{b}}, \Delta t$ is a characteristic life time of alphas with the energy $\mathcal{E}_{\alpha}, \tau_{\mathrm{b}} \ll \Delta t \ll \tau_{\mathrm{s}}, v_{\alpha}=\sqrt{2 \mathcal{E}_{\alpha} / M_{\alpha}}$, the integral is taken along particle orbits, so that $F_{\alpha}$ is determined by the drift constants of motion (COM). Usually one uses the particle canonical angular momentum $\left(P_{\phi}\right)$, the energy $(\mathcal{E})$, the magnetic moment $\left(\mu_{p}\right)$, and direction of the longitudinal motion of passing particles $\left(\sigma=\operatorname{sgn} v_{\|}\right)$in order to describe the motion of particle guiding center. It is more convenient, however, to use another set of COMs for the description of distribution of confined particles. Namely, the maximum radial displacement during the orbital motion $\left(r_{\max }\right)$ instead of $P_{\phi}$ and the pitch parameter $\lambda=\mu_{p} \bar{B} / \mathcal{E}(\bar{B}$ is the magnetic field at the magnetic axis). It is clear that confined are those particles which have $r_{\max }<a$, toroidally trapped are the particles with $b_{\max }^{-1}<\lambda<b_{\min }^{-1}\left(b \equiv B(r, \vartheta) / \bar{B}, b_{\max }\right.$ and $b_{\min }$ are maximum and minimum $b$ on the orbit) and passing are the particles with $0 \leqslant \lambda<b_{\max }^{-1}$.

With these COMs, we can write:

$$
\left\langle S_{\alpha}\right\rangle=\left\{\oint \frac{\mathrm{d} \vartheta S_{\alpha}[r(\vartheta)]}{v_{\|}}\right\} \times\left\{\oint \frac{\mathrm{d} \vartheta}{v_{\|}}\right\}^{-1},
$$

$r(\vartheta)=r\left[r_{\max }, \lambda, v_{\alpha}, \vartheta\right], \quad v_{\|}= \pm v \sqrt{1-\lambda b(r, \vartheta)}$. Here the integrals are taken over the whole $\vartheta$ region, $(0,2 \pi)$, for passing particles and between the turning points for trapped particles (with taking into account that the sign of $v_{\|}$changes after passing the turning points).

In the peripheral region, which we are interested in, the approximation of narrow orbit width $(\Delta \ll r$, with $\Delta$ the orbit half width) is justified. The corresponding condition reads: $r>R\left(q \rho / \kappa_{E} R\right)^{2 / 3}\left(\kappa_{E}\right.$ is the plasma elongation, $\rho=v_{\alpha} / \omega_{B \alpha}$ ), which for alphas in JET yields $r>20 \mathrm{~cm}$.

The majority of passing particles have $v_{\|} \approx$ const. Due to this, we can describe their orbits as follows:

$$
r^{\text {pas }}(\vartheta)=r_{\text {max }}-\Delta_{p}+\sigma \Delta_{p} \cos \vartheta,
$$

$\Delta_{p}=q\left(r_{0}\right) \rho \sqrt{1-\lambda} / \kappa_{E}$, the subscript ' $p$ ' labels passing orbits. In this approximation, when calculating integrals in equation (B.3), we have to put $b(r, \vartheta)=1$ and $0 \leqslant \vartheta \leqslant 2 \pi$.

The orbits of trapped particles are given by

$$
\begin{array}{r}
r_{1,2}^{\mathrm{tr}}(\vartheta)=r_{0}+\frac{q \rho}{\kappa_{E} \sqrt{\epsilon_{0}}}[ \\
{\left[-\sigma_{0} \sqrt{\frac{1-\lambda}{\epsilon_{0}}+\lambda \cos \vartheta_{0}}\right.} \\
\left. \pm \sqrt{\frac{1-\lambda}{\epsilon_{0}}+\lambda \cos \vartheta}\right],
\end{array}
$$

where $\epsilon=r / R$, the subscript ' 0 ' labels a point on the orbit, the particle turning points $\left(\vartheta_{t}\right)$ are determined by

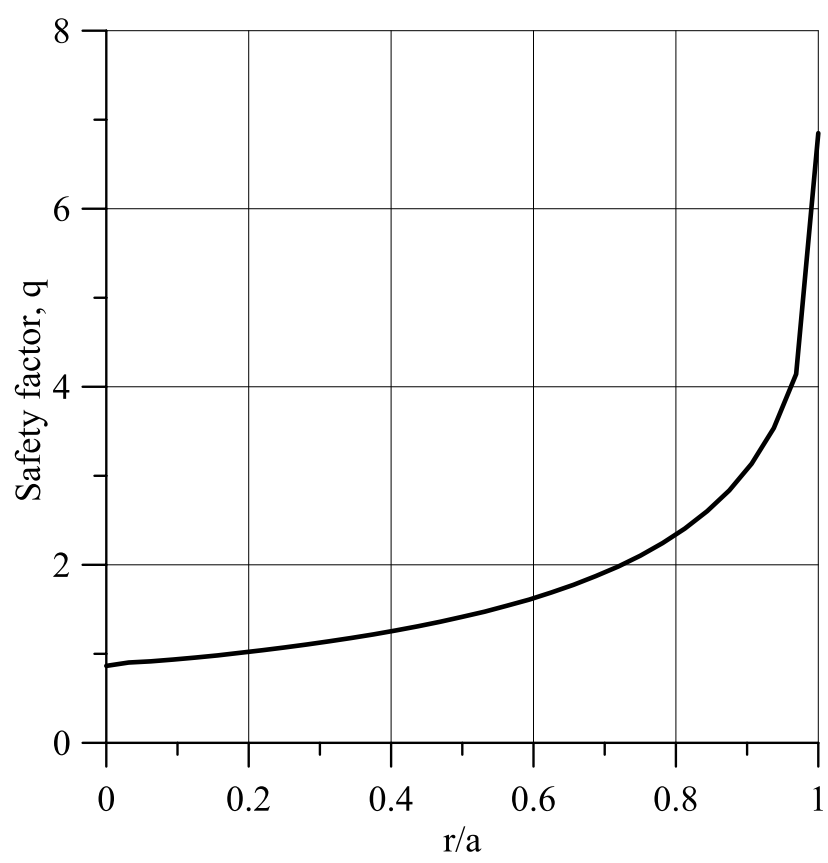

Figure B3. The safety factor in discharge \#42856.

$\cos \vartheta_{t}=\left(1-\lambda^{-1}\right) / \epsilon_{0}$. When calculating integrals in (B.3), both branches given by (B.5) should be taken into account. It is convenient to proceed to the angle $\beta$ defined by $\sin ^{2}(\vartheta / 2)=\kappa_{p}^{2} \sin ^{2} \beta$, with $\kappa_{p}^{2}=\left[0.5+\left(\lambda^{-1}-1\right) /\left(2 \epsilon_{0}\right)\right]>0$ is the particle trapping parameter $\left[\kappa_{p}^{2}<1\right.$ for trapped particles, which implies $\left.\lambda>(1+\epsilon)^{-1}\right], \sin \beta_{0}=\kappa_{p}^{-1} \sin \left(\vartheta_{0} / 2\right)$. Then

$$
r_{1,2}^{\mathrm{tr}}(\beta)=r_{\max }-\Delta_{t}+\sigma \Delta_{t} \cos \beta,
$$

$\Delta_{t}=q(\bar{r}) \rho\left(\kappa_{p} / \kappa_{E}\right) \sqrt{2 \lambda / \bar{\epsilon}}, \bar{r}=r_{\max }-\Delta_{t}$. In these variables

$$
\begin{aligned}
\left\langle S_{\alpha}\right\rangle^{\mathrm{tr}}= & \frac{1}{2}\left\{\sum_{j=1,2} \int_{0}^{\pi / 2} \frac{\mathrm{d} \beta S_{\alpha}\left[r_{j}^{\mathrm{tr}}(\beta)\right]}{\sqrt{1-\kappa_{p}^{2} \sin ^{2} \beta}}\right\} \\
& \times\left\{\int_{0}^{\pi / 2} \frac{\mathrm{d} \beta}{\sqrt{1-\kappa_{p}^{2} \sin ^{2} \beta}}\right\}^{-1} .
\end{aligned}
$$

If the alpha source function is approximated as $S_{\alpha}[r(\vartheta)]=S_{m}+S_{m}^{\prime}\left(r-r_{\max }\right)\left[S_{m} \equiv S_{\alpha}\left(r_{\max }\right), S_{m}^{\prime} \equiv S_{\alpha}^{\prime}\left(r_{\max }\right)\right.$, and prime denotes the radial derivative] we obtain for passing particles:

$$
\left\langle S_{\alpha}\right\rangle\left(r_{\max }\right)=S_{m}-S_{m}^{\prime} \Delta_{p} .
$$

A similar equation is true for deeply trapped particles (particles with $\left.\kappa_{p} \ll 1\right)$. Equation (B.8) shows that $\left\langle S_{\alpha}\right\rangle\left(r_{\max }=a\right) \neq 0$ even when the birth source vanishes at the plasma edge, $S_{m}=0$. On the other hand, $\left\langle S_{\alpha}\right\rangle=0$ in the near-axis region $\left[r \lesssim R\left(q \rho / \kappa_{E} R\right)^{2 / 3}\right.$ for $\lambda \sim 1$ and $r<2 q \rho$ for well passing particles] because there are no particles with smaller $r_{\text {max }}$. This implies that the distribution of $\left\langle S_{\alpha}\right\rangle\left(r_{\max }\right)$ is shifted outwards with respect to $S_{\alpha}(r)$, increasing the number of alpha particles which can interact with the waves in the peripheral region.

To be more specific, we carried out numerical calculations for trapped and passing alphas in discharge \#42856. 


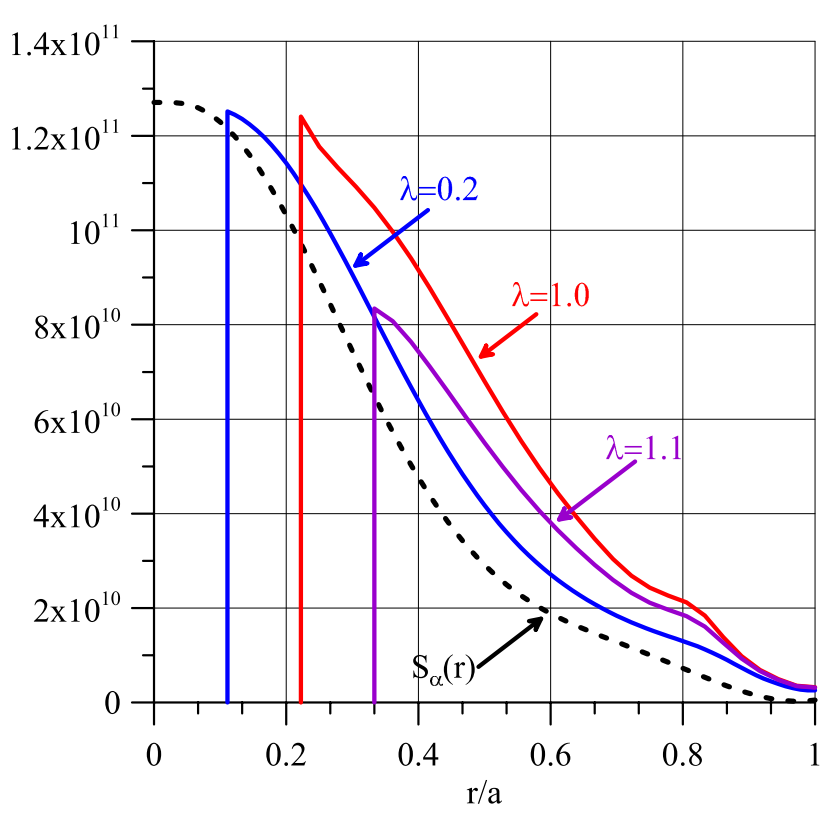

Figure B4. Bounce/transit averaged source function $\left\langle S_{\alpha}\right\rangle\left(r_{\max }, \lambda, \mathcal{E}_{\alpha}\right)$ versus $r$ for various $\lambda$ in discharge \#42856. This picture demonstrates that the radial distribution of the density of alpha particles with given $r_{\max }\left[n_{\alpha}\left(r_{\max }\right) \propto\left\langle S_{\alpha}\right\rangle\left(r_{\max }\right)\right]$ is considerably shifted outwards in comparison with the alpha birth profile (shown by dashed line).

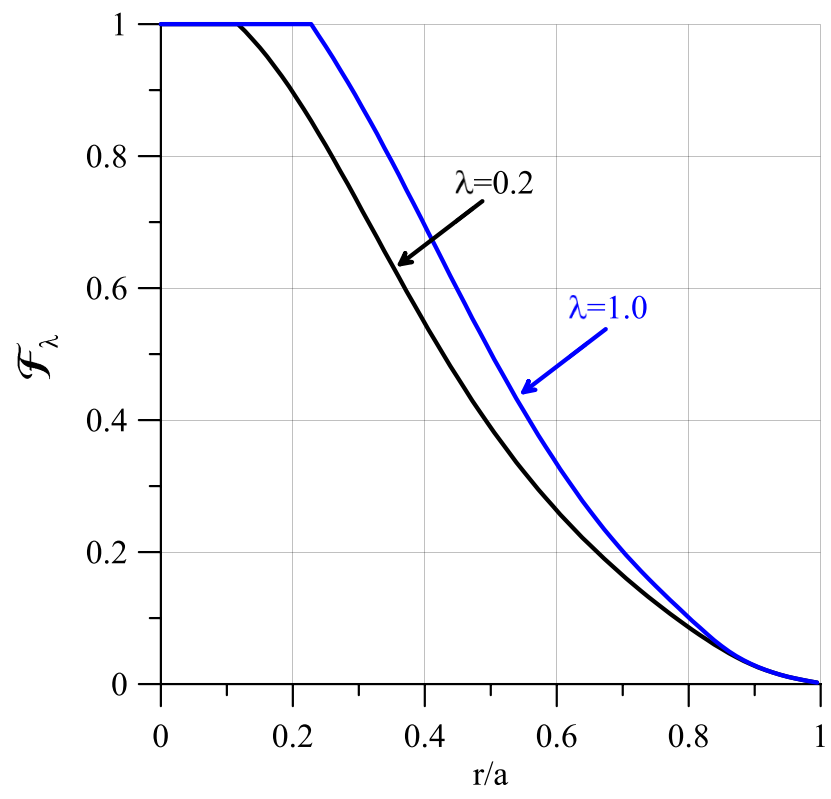

Figure B5. Fraction of passing alpha particles $(\lambda=0.2, \sigma=1$ or $\sigma=-1)$ and trapped particles $(\lambda=1)$ that have $r_{\max }$ outside a certain radius $r$ in discharge \#42856.

The results obtained with using the safety factor $q(r)$ given by figure B3 are shown in figure B4.

The fraction of $3.5 \mathrm{MeV}$ alpha particles with given $\lambda$ and $r_{\text {max }}$ outside a certain radius $r$ is described by

$$
\mathcal{F}_{\lambda}(r) \equiv \frac{\int_{r}^{a} \mathrm{~d} r_{\max } r_{\max }\left\langle S_{\alpha}\right\rangle\left(r_{\max }, \lambda\right)}{\int_{0}^{a} \mathrm{~d} r_{\max } r_{\max }\left\langle S_{\alpha}\right\rangle\left(r_{\max }, \lambda\right)}
$$

and presented in figure B5. We observe that at the periphery $\mathcal{F}_{\lambda}$ exceeds $\mathcal{F}^{(0)}$ given by (B.2), the effect being considerable even for passing particles.

\section{Appendix C. Damping of high-frequency fast magnetoacoustic waves}

In this appendix we study the electron and ion damping of the high-frequency fast magnetoacoustic waves (FMW) for which both the electrons and thermal ions can be treated as cold particles. The purpose of this consideration is to see whether the bulk plasma particles can lead to the damping rate which is comparable to the FMW drive produced by alpha particles. This implies that we are interested in the FMW which satisfy the following conditions:

$$
\omega \approx l \omega_{B i} \gg \omega_{B i},
$$

$\left(\omega-l \omega_{B i}\right)^{2} \gg\left(k_{\|} v_{T i}\right)^{2}, \quad \omega^{2} \gg\left(k_{\|} v_{T e}\right)^{2}, \quad k_{\perp} \rho_{i}<1$,

where $\rho_{i}=v_{T i} / \omega_{B i}, k_{\perp}$ is the wavenumber across the magnetic field.

Assuming that the perturbations of the electromagnetic field are proportional to $\exp \left(-\mathrm{i} \omega t+\mathrm{i} k_{x} x+\mathrm{i} k_{z} z\right)$, with the $z$-axis directed along the magnetic field, we proceed from the Maxwell equations $\boldsymbol{B}=(c / \omega) \boldsymbol{k} \times \boldsymbol{E}$ and $\boldsymbol{k} \times \boldsymbol{B}=-(\omega / c) \boldsymbol{\varepsilon} \cdot \boldsymbol{E}$, with $\varepsilon \equiv \varepsilon_{i j}$ the dielectric tensor. Then, taking into account equation (C.2), we can obtain the following equations:

$$
\begin{gathered}
\left(\varepsilon_{1}-\frac{N_{\|}^{2}}{1-N_{x}^{2} / \eta}\right) E_{x}+\mathrm{i} g E_{y}=0 . \\
-\mathrm{i} g E_{x}+\left(\varepsilon_{2}-N^{2}\right) E_{y}=0,
\end{gathered}
$$

where $\varepsilon_{1}=\varepsilon_{x x}, \varepsilon_{2}=\varepsilon_{y y}, \eta=\varepsilon_{z z}$, and $g$ is defined by $\varepsilon_{x y}=\mathrm{i} g, N^{2}=c^{2} k^{2} / \omega^{2}, N_{\|}^{2}=c^{2} k_{\|}^{2} / \omega^{2}$, and $N_{x}^{2}=c^{2} k_{x}^{2} / \omega^{2}$. These equations lead to the dispersion relation given by

$\Lambda(\omega) \equiv\left(\varepsilon_{1}-\frac{N_{\|}^{2}}{1-N_{x}^{2} / \eta}\right)\left(\varepsilon_{2}-N^{2}\right)-g^{2}=0$.

When $\operatorname{Im} \varepsilon_{i j} \ll \operatorname{Re} \varepsilon_{i j}$, equation (C.5) can be solved perturbatively. Taking $\omega=\omega_{0}+\mathrm{i} \gamma$, with $\omega_{0}$ mode frequency and $\gamma \ll \omega_{0}$ the damping rate, we can write $\Lambda(\omega)=\Lambda_{0}\left(\omega_{0}\right)+\mathrm{i} \gamma \mathrm{d} \Lambda / \mathrm{d} \omega_{0}+\mathrm{i} \lambda\left(\omega_{0}\right)=0$. Then $\omega_{0}$ is a solution of the equation $\Lambda_{0}=0$ and the damping rate is given by

$$
\gamma=-\left.\frac{\lambda}{\mathrm{d} \Lambda / \mathrm{d} \omega}\right|_{\omega_{0}}
$$

To calculate $\gamma$ by means of this equation we have to specify components of the dielectric tensor.

Proceeding from the well-known general expressions for the dielectric tensor of a Maxwellian plasma and using (C.2) with $\omega \ll \omega_{B e}$, we obtain:

$$
\begin{aligned}
\varepsilon_{1}= & -\frac{\omega_{p i}^{2}}{\omega^{2}}+\mathrm{i} \sqrt{\pi} \frac{\omega_{p i}^{2}}{\omega k_{\|} v_{T i}} \sum_{n} \mathrm{e}^{-x_{n}^{2}} \frac{n^{2}}{z} \mathrm{e}^{-z} I_{n} . \\
\varepsilon_{2}= & \varepsilon_{1}+\mathrm{i} \sqrt{\pi} \frac{\omega_{p i}^{2}}{\omega k_{\|} v_{T i}} \sum_{n} \mathrm{e}^{-x_{n}^{2}} 2 z \mathrm{e}^{-z}\left(I_{n}-I_{n}^{\prime}\right) \\
& +\mathrm{i} \sqrt{\pi} \frac{\omega_{p e}^{2}}{\omega^{2}} k_{\perp}^{2} \rho_{e}^{2} x_{e} \mathrm{e}^{-x_{e}^{2}},
\end{aligned}
$$




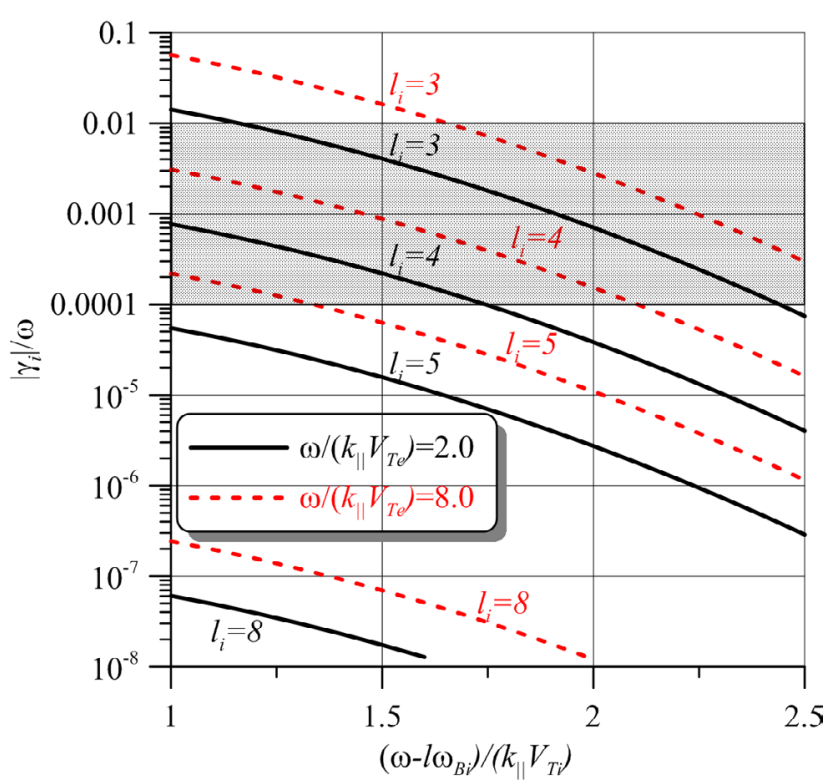

Figure $\mathbf{C}$. Ion damping rate. The hatched region is of main interest because the instability drive is expected to be $\gamma_{\alpha} / \omega=10^{-4}-10^{-2}$. $l_{i}=l_{\alpha}-1$.

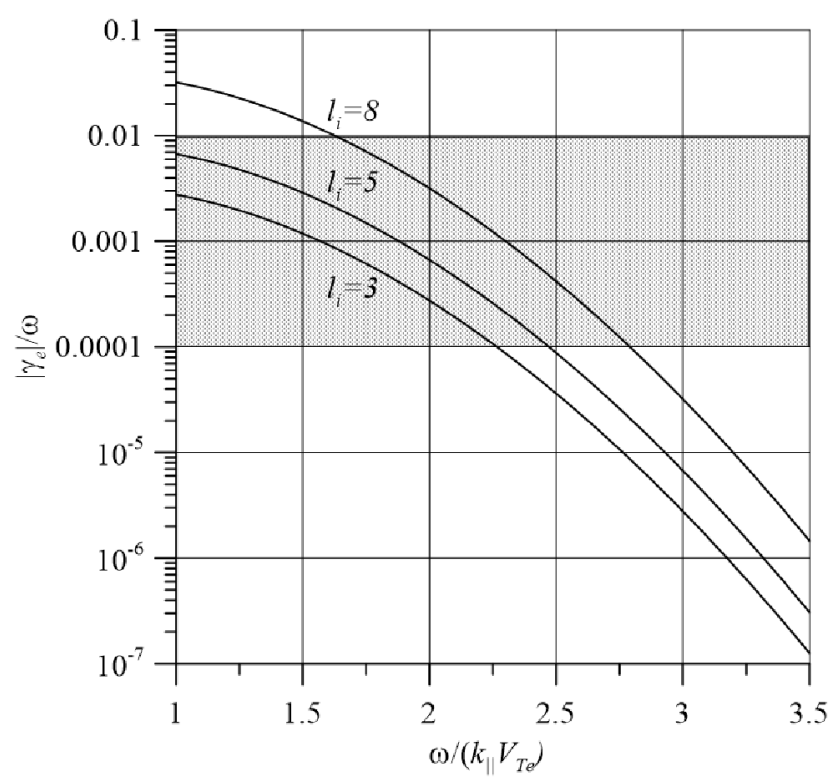

Figure C2. Electron damping rate. The hatched region is of main interest because the instability drive is expected to be $\gamma_{\alpha} / \omega=10^{-4}-10^{-2}$.

$g=-\frac{\omega_{p i}^{2}}{\omega \omega_{B i}}+\mathrm{i} \sqrt{\pi} \frac{\omega_{p i}^{2}}{\omega k_{\|} v_{T i}} \sum_{n} \mathrm{e}^{-x_{n}^{2}} \mathrm{e}^{-z} n\left(I_{n}-I_{n}^{\prime}\right)$.

$\eta=-\frac{\omega_{p e}^{2}}{\omega^{2}}+2 \mathrm{i} \sqrt{\pi}\left(\frac{\omega_{p i}^{2}}{\omega k_{\|} v_{T i}} \sum_{n} x_{n}^{2} \mathrm{e}^{-x_{n}^{2}-z}+\frac{\omega_{p e}^{2}}{k_{\|}^{2} v_{T e}^{2}} x_{e} \mathrm{e}^{-x_{e}^{2}}\right)$,

where $\quad \omega_{p e, i}^{2}=4 \pi n_{e, i}^{2} / M_{e, i}, \quad I_{n}=I_{n}(z) \quad$ is the modified Bessel function on the $n$th order, $z=k_{\perp}^{2} \rho_{i}^{2} / 2$, $x_{n}=\left(\omega-n \omega_{B i}\right) /\left(k_{\|} v_{T i}\right), x_{e}=\omega /\left(k_{\|} v_{T e}\right)$.

As a result, we have $\omega^{2}=k^{2} v_{A}^{2}\left(1+k_{\|}^{2} v_{\|}^{2} / \omega_{B i}^{2}\right)$ and

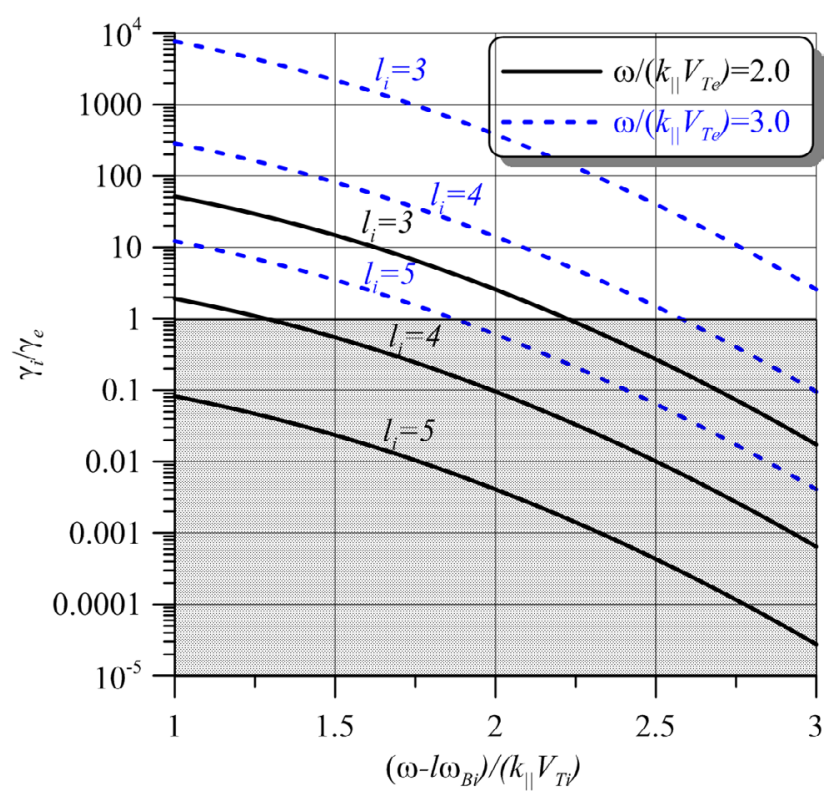

Figure C3. Ratio of the ion damping rate to the electron damping rate. Hatched is the region with dominant electron damping.

$$
\begin{array}{r}
\frac{\gamma_{i}}{\omega}=-\frac{\sqrt{\pi}}{2} \frac{k v_{A}}{\mid k_{\|} v_{T i}} \sum_{n} \mathrm{e}^{-x_{n}^{2}} \frac{n^{2}}{z} \mathrm{e}^{-z} I_{n}, \\
\frac{\gamma_{e}}{\omega}=-\sqrt{\pi} x_{e} \mathrm{e}^{-x_{e}^{2}} \frac{M_{e}}{M_{i}}\left(\frac{k_{\perp}^{2} k^{2} v_{A}^{4}}{\omega_{B i}^{4}} \frac{v_{A}^{2}}{v_{T e}^{2}}+\frac{k_{\perp}^{2}}{2 k^{2}} \frac{v_{T e}^{2}}{v_{A}^{2}}\right) .
\end{array}
$$

Here the subscript ' 0 ' at $\omega$ is omitted.

The ion damping rate and the electron damping rate are shown in figures $\mathrm{C} 1-\mathrm{C} 3$ for the following parameters: $T_{i}=15 \mathrm{keV}, T_{e}=10 \mathrm{keV}, n_{e}=4 \times 10^{13} \mathrm{~cm}^{-3}, B=3.5 \mathrm{~T}$.

\section{References}

[1] Thomas P.R. et al 1998 Phys. Rev. Lett. 805548

[2] Thomas P.R. et al 2001 Proc. 28th EPS Conf. Controlled Fusion and Plasma Physics Europhysics Conf. Abstracts (Madeira, Portugal, 18-22 June 2001) vol 25A (EPS) p 929 (www.cfn.ist.utl.pt/EPS2001/fin/index.html)

[3] Sharapov S.E. et al 2008 Fusion Sci. Technol. 53989

[4] Weisen H. et al 2014 AIP Conf. Proc. 161277

[5] Kolesnichenko Ya.I., Yakovenko Yu.V. and Lutsenko V.V. 2010 Phys. Rev. Lett. 10407500

[6] Kolesnichenko Ya.I., Yakovenko Yu.V., Lutsenko V.V., Weller A. and White R.B. 2010 Nucl. Fusion 50084011

[7] Stutman D. et al 2009 Phys. Rev. Lett. 102115002

[8] Gorelenkov N.N. et al 2010 Nucl. Fusion 50084012

[9] Crocker N. et al 2017 15th IAEA Technical Meeting on Energetic Particles in Magnetic Confinement Systems Programme, Abstracts (Princeton, USA, 5-8 September 2017) (Vienna: IAEA) p 18 (https://nucleus.iaea.org/sites/fusionportal/ Pages/Energetic\%20Particles\%2017/Presentations.aspx)

[10] Cottrell G.A. and Dendy R.O. 1988 Phys. Rev. Lett. 6033

[11] Cauffman S. and Majeski R. 1995 Rev. Sci. Instrum. 66817

[12] Ichimura M. et al 2008 Nucl. Fusion 48035012

[13] Saito K. et al 2013 Plasma Sci. Technol. 15209

[14] Heidbrink W.W. et al 2011 Plasma Phys. Control. Fusion 53085028 
[15] D'Inca R. et al 2011 Proc. 38th EPS Conf. Plasma Phys. (Strasbourg, France, 27 June-1 July 2011) P1.053 (http://ocs.ciemat.es/EPS2011PAP/html/contrib.html)

[16] Cottrell G.A. et al 1993 Nucl. Fusion 331365

[17] Cottrell G.A. et al 2000 Phys. Rev. Lett. 842397

[18] McClements K.G. et al 2015 Nucl. Fusion 55043013

[19] Dendy R.O. et al 1995 Nucl. Fusion 351733

[20] Gorelenkov N.N. and Cheng C.Z. 1995 Nucl. Fusion 351743

[21] Kolesnichenko Ya.I., Anderson D., Fülöp T. and Lisak M. 1997 Proc. 16th IAEA Conf. of Fusion Energy (Montreal, Canada, 7-11 October 1996) vol 2 (Vienna: IAEA) 487 (http://iaea.org/inis/collection/NCLCollectionStore/_ Public/29/009/29009147.pdf?r=1)

[22] Fülöp T., Kolesnichenko Ya.I., Lisak M. and Anderson D. 1997 Nucl. Fusion 271281

[23] Gorelenkov N.N., Pinches S.D. and Toi K. 2014 Nucl. Fusion 54125001

[24] Dendy R.O., Lashmore-Davies C.N. and Kam K.F. 1992 Phys. Fluids B 43996

[25] Crocker N. et al 2013 Nucl. Fusion 53043017

[26] Smith H.M. and Verwichte E. 2009 Plasma Phys. Control. Fusion $\mathbf{5 1} 075001$
[27] Kolesnichenko Ya.I., Fülöp T., Lisak M. and Anderson D. 1998 Nucl. Fusion 381871

[28] Sigmar D.J. and Joyce G. 1971 Nucl. Fusion 11447

[29] Cook J.W.S., Dendy R.O. and Chapman S.C. 2017 Phys. Rev. Lett. 118185001

[30] Tyshchenko M.H. and Yakovenko Yu.V. 2015 Probl. At. Sci. Technol. 149 (http://dspace.nbuv.gov.ua/bitstream/ handle/123456789/82065/12-Tyshchenko.pdf?sequence=1)

[31] Testa D. and Albergante M. 2012 Europhys. Lett. 9735003

[32] Garcia J. et al 2015 Nucl. Fusion 55053007

[33] Hyun-Tae K. et al 2018 Nucl. Fusion 58036020

[34] Budny R.V. and JET Contributors 2016 Nucl. Fusion 56036013

[35] Jacquinot J. and JET Team 1998 Nucl. Fusion 381263

[36] Maggi C.F. et al 2018 Plasma Phys. Control. Fusion 60014045

[37] Urano H. et al 2013 Nucl. Fusion 53083003

[38] White R.B. 2001 The Theory of Toroidally Confined Plasmas (London: Imperial College Press) p 295

[39] Kolesnichenko Ya.I. and Yavorskij V.A. 1989 Nucl. Fusion 291319

[40] Litaudon X. et al 2017 Nucl. Fusion 57102001 